Journal of Algebraic Combinatorics 16 (2002), 43-69 c) 2002 Kluwer Academic Publishers. Manufactured in The Netherlands.

\title{
Transitive Permutation Groups of Prime-Squared Degree
}

EDWARD DOBSON

dobson@math.msstate.edu

Department of Mathematics and Statistics, Mississippi State University, Mississippi State, MS 39762, USA

DAVE WITTE

dwitte@math.okstate.edu

Department of Mathematics, Oklahoma State University, Stillwater, OK 74078, USA

Received November 7, 2000; Revised November 29, 2001

\begin{abstract}
We explicitly determine all of the transitive groups of degree $p^{2}, p$ a prime, whose Sylow $p$-subgroup is not isomorphic to the wreath product $\mathbb{Z}_{p} \imath \mathbb{Z}_{p}$. Furthermore, we provide a general description of the transitive groups of degree $p^{2}$ whose Sylow $p$-subgroup is isomorphic to $\mathbb{Z}_{p} 2 \mathbb{Z}_{p}$, and explicitly determine most of them. As applications, we solve the Cayley Isomorphism problem for Cayley objects of an abelian group of order $p^{2}$, explicitly determine the full automorphism group of Cayley graphs of abelian groups of order $p^{2}$, and find all nonnormal Cayley graphs of order $p^{2}$.
\end{abstract}

Keywords: permutation group, Cayley graph, $p$-group

\section{Introduction}

In 1901, Burnside [5] proved the following theorem.

Theorem 1 (Burnside, [5]) Let $G$ be a transitive group of prime degree. Then either $G$ is doubly transitive or $G$ contains a normal Sylow p-subgroup.

If $G$ is a transitive group of prime degree and has a normal Sylow $p$-subgroup, then it is not difficult to show that $G$ is permutation isomorphic to a subgroup of $\operatorname{AGL}(1, p)$. Similarly, it is also straightforward to show that if $G$ is a transitive group of prime degree, then $G$ has a normal Sylow $p$-subgroup if and only if $G$ is solvable.

A well-known consequence of the classification of the finite simple groups is that all doubly transitive groups are known [8, Theorem 5.3], and hence all doubly transitive groups of prime degree are known.

Combining these results yields the following well-known classification of all transitive groups of prime degree.

Definition 1 We use the following standard notation.

- $S_{p}$ and $A_{p}$, respectively, denote a symmetric group and an alternating group of degree $p$,

- $\operatorname{AGL}(d, p)=\mathbb{Z}_{p}^{d} \rtimes \mathrm{GL}(d, p)$ denotes the group of affine transformations of the $d$ dimensional vector space $\mathbb{F}_{p}^{d}$ over $\mathbb{F}_{p}$, 
- $M_{11}$ and $M_{23}$ denote Mathieu groups,

- $\operatorname{PSL}(d, q)$ and $\operatorname{PGL}(d, q)$, respectively, denote a projective special linear group and a projective general linear group over the field $\mathbb{F}_{q}$ of $q$ elements, and

- $\operatorname{P\Gamma L}(d, q)$ denotes the semidirect product of $\operatorname{PGL}(d, q)$ with the group of Galois automorphisms of $\mathbb{F}_{q}$.

Theorem 2 ([12, Corollary 4.2]) Suppose $H$ is a subgroup of $S_{p}$ that contains $\mathbb{Z}_{p}$. Let $S$ be a minimal normal subgroup of $H$, and let $N=N_{S_{p}}(S)$, so $S$ is simple and $S \leq H \leq N$. Then $N / S$ is cyclic, and either:

1. $S=\mathbb{Z}_{p}, N=\operatorname{AGL}(1, p)$, and $N / S \cong \mathbb{Z}_{p-1}$; or

2. $S=A_{p}, N=S_{p}$, and $N / S \cong \mathbb{Z}_{2} ;$ or

3. $p=11$ and $S=H=N=\operatorname{PSL}(2,11)$; or

4. $p=11$ and $S=H=N=M_{11}$; or

5. $p=23$ and $S=H=N=M_{23}$; or

6. $p=\left(r^{d^{m+1}}-1\right) /\left(r^{d^{m}}-1\right)$ for some prime $r$ and natural numbers $d$ and $m$, and we have $S=\operatorname{PSL}\left(d, r^{d^{m}}\right), N=\operatorname{P\Gamma L}\left(d, r^{d^{m}}\right)$, and $N / S \cong \mathbb{Z}_{m}$.

In this paper, we will begin the classification of all transitive groups of degree $p^{2}$. Our starting point is Theorem 3 below (proved at the end of Section 3), which provides an analogue of Burnside's Theorem 1. This allows us to determine all of the transitive permutation groups of degree $p^{2}$ that do not have Sylow $p$-subgroup isomorphic to the wreath product $\mathbb{Z}_{p} \geq \mathbb{Z}_{p}$ (see Theorem 4; the proof appears at the end of Section 4). Furthermore, Proposition 1 below describes how to construct every imprimitive permutation group of degree $p^{2}$ whose Sylow $p$-subgroup is isomorphic to $\mathbb{Z}_{p} 2 \mathbb{Z}_{p}$. (This proposition is proved at the beginning of Section 5.) Unfortunately, this proposition does not provide a complete classification of these permutation groups, because, in some cases, we do not have an explicit description of the possible choices for $K$ and $\phi$ in the conclusion of the proposition. However, Theorem 2 describes the possible choices for $H$ and $L$, and, in most cases, Section 5.1 describes the possible choices for $K$, and Section 5.2 describes the possible choices for $\phi$. This leads to a complete classification for most primes $p$; specifically, the classification is complete for any prime $p$, such that $p \notin\{11,23\}$ and $p \neq\left(q^{d}-1\right) /(q-1)$, for every prime-power $q$ and natural number $d$. The problems that remain are described in a remark at the end of Section 5.

Definition 2 (cf. Definition 5) Let $P_{p-1}^{\prime}$ denote the unique subgroup of $S_{p^{2}}$ (up to conjugacy) having order $p^{p}$ and containing a transitive subgroup isomorphic to $\mathbb{Z}_{p} \times \mathbb{Z}_{p}$ (and therefore not containing a transitive cyclic subgroup; see Lemma 4).

Theorem 3 Let $G$ be a transitive permutation group of degree $p^{2}$, $p$ a prime, with Sylow $p$-subgroup $P$. Then either

1. $G$ is doubly transitive; or

2. $P \triangleleft G ;$ or

3. $P$ is equivalent to either $\mathbb{Z}_{p} \times \mathbb{Z}_{p}, P_{p-1}^{\prime}$, or $\mathbb{Z}_{p} 2 \mathbb{Z}_{p}$. 
Theorem 4 Let $G$ be a transitive group of degree $p^{2}$ such that a Sylow p-subgroup $P$ of $G$ is not isomorphic to $\mathbb{Z}_{p} 2 \mathbb{Z}_{p}$. Then, after replacing $G$ by a conjugate, one of the following is true.

1. $G$ is doubly transitive, and either

- $G=A_{p^{2}}$ or $S_{p^{2}} ;$ or

- $\operatorname{PSL}(d, q) \leq G \leq \operatorname{P\Gamma L}(d, q)$, where $\left(q^{n}-1\right) /(q-1)=p^{2} ;$ or

- $\mathbb{Z}_{p} \times \mathbb{Z}_{p} \leq G \leq \operatorname{AGL}(2, p)$.

2. $G$ is simply primitive, has an elementary abelian Sylow p-subgroup and either

- $\mathbb{Z}_{p} \times \mathbb{Z}_{p} \leq G \leq \operatorname{AGL}(2, p)$; or

- $G$ has a transitive, imprimitive subgroup $H$ of index 2 , such that $H \leq S_{p} \times S_{p}$ (so $H$ is described in Lemma 1$)$,

3. $G$ is imprimitive, $P ¥ \mathbb{Z}_{p} \times \mathbb{Z}_{p}, P ¥ P_{p-1}^{\prime}$, and $P \triangleleft G$, so $P \leq G \leq N_{S_{p^{2}}}(P$ ) (and $N_{S_{p^{2}}}(P)$ is described in Lemma 5 or 6$)$;

4. $G$ is imprimitive, $P=\mathbb{Z}_{p} \times \mathbb{Z}_{p}$ and $G \leq S_{p} \times S_{p}$ (so $G$ is described in Lemma 1 ); or

5. $G$ is imprimitive, $P=P_{p-1}^{\prime}$, and $G=L P$, where $\mathbb{Z}_{p} \times \mathbb{Z}_{p} \leq L \leq S_{p} \times \operatorname{AGL}(1, p)$ (so $L$ is described in Lemma 1).

Definition 3 ([9, p. 168]) Let $H$ be a group and let $A$ be an $H$-module. (That is, $A$ is an abelian group on which $H$ acts by automorphisms. Also note that abelian groups, when viewed as modules, are written additively.) A function $\phi: H \rightarrow A$ is a crossed homomorphism if, for every $h_{1}, h_{2} \in H$, we have

$$
\phi\left(h_{1} h_{2}\right)=h_{2}^{-1} \cdot \phi\left(h_{1}\right)+\phi\left(h_{2}\right) .
$$

(This is equivalent to the assertion that the function $H \rightarrow H \ltimes A$ defined by $h \mapsto(h, \phi(h))$ is a homomorphism.)

\section{Proposition 1 Let}

1. $p$ be a prime;

2. $H$ and $L$ be transitive subgroups of $S_{p}$, such that $L$ is simple;

3. $K$ be an $H$-invariant subgroup of the direct product $\left(N_{S_{p}}(L)\right)^{p}$ containing $L^{p}$;

4. $\phi: H \rightarrow N_{S_{p}}(L)^{p} / K$ be a crossed homomorphism; and

5. $G_{H, L, K, \phi}=\left\{(h, v) \in H \ltimes N_{S_{p}}(L)^{p}: \phi(h)=v K\right\} \leq S_{p} 2 S_{p}$.

Then $G_{H, L, K, \phi}$ is a transitive, imprimitive subgroup of $S_{p^{2}}$, such that a Sylow p-subgroup of $G$ is isomorphic to $\mathbb{Z}_{p} \geq \mathbb{Z}_{p}$.

Conversely, if $G$ is a transitive, imprimitive permutation group of degree $p^{2}$, such that a Sylow p-subgroup of $G$ is isomorphic to $\mathbb{Z}_{p} 2 \mathbb{Z}_{p}$, then $G$ is equivalent to $G_{H, L, K, \phi}$, for some $H, L, K$, and $\phi$ as above.

To some extent, our proofs follow the outline that was used to determine all transitive groups of prime degree.

In Section 2, we recall known results that provide a classification of certain types of transitive permutation groups of degree $p^{2}$, namely, doubly transitive groups, groups with elementary abelian Sylow $p$-subgroup, and simply primitive groups. (Recall that a permutation group is simply primitive if it is primitive, but not doubly transitive.) 
In Section 3, we extend Theorem 1 to transitive groups of degree $p^{2}$. It follows by [33, Theorem $3.4^{\prime}$ ] that every transitive group of prime power degree contains a transitive Sylow $p$-subgroup; in particular, every transitive group of degree $p^{2}$ contains a transitive Sylow $p$-subgroup. We first show that there are exactly $2 p-1$ transitive $p$-subgroups of $S_{p^{2}}$ up to permutation isomorphism and explicitly determine them (see Theorem 9). We also calculate the normalizer of each of these $p$-subgroups (see Lemmas 5 and 6). Next, we prove Theorem 3, which extends Burnside's Theorem 1 to transitive groups of degree $p^{2}$; that is, it determines which of these $2 p-1 p$-subgroups $P$ have the property that if $G \leq S_{p^{2}}$ with Sylow $p$-subgroup $P$, then either $P \triangleleft G$ or $G$ is doubly transitive. Happily, only three of the $2 p-1$ transitive $p$-subgroups of $S_{p^{2}}$ fail to have this property.

We are left with the problem of finding every imprimitive or simply primitive subgroup of $S_{p^{2}}$ whose Sylow $p$-subgroup is one of the three transitive $p$-subgroups of $S_{p^{2}}$ for which the extension of Burnside's Theorem mentioned above does not hold. Two of these $p$-subgroups are $\mathbb{Z}_{p}^{2}$ and the group $P_{p-1}^{\prime}$ (see Definition 2 or 5), which can, in a natural way, be regarded as the "dual" of $\mathbb{Z}_{p}^{2}$. These two $p$-subgroups are considered in Section 4 , and the remaining $p$-subgroup, $\mathbb{Z}_{p} 2 \mathbb{Z}_{p}$, is considered in Section 5. However, as explained in the comments before Definition 2 , our results on $\mathbb{Z}_{p} 2 \mathbb{Z}_{p}$ are not complete.

In Section 6, we prove some straightforward applications of the above results that are of interest to combinatorialists.

We remark that some of the intermediate results (as well as some of the applications) in this paper are known, and will give appropriate references as needed.

\section{Some known results}

\subsection{Doubly transitive groups}

The doubly transitive groups of degree $p^{2}$ can be determined much as in the case of degree $p$. Burnside [6, p. 202] proved the following result.

Theorem 5 (Burnside, [6]) The socle of a finite doubly transitive group is either a regular elementary abelian p-group, or a nonregular nonabelian simple group.

If $G$ is doubly transitive of degree $p^{2}$, then it is not difficult to show that the socle of $G$ is abelian if and only if $G \leq \operatorname{AGL}(2, p)$. (Note that an elementary abelian group of order $p^{2}$ is isomorphic to $\mathbb{Z}_{p}^{2}$. Also, we remark that the doubly transitive subgroups of $\operatorname{AGL}(d, p)$ have been determined [16, 20], cf. [8, proof of Theorem 5.3]).

The doubly transitive groups with nonabelian socle are listed in [8, Table on p. 8]. (This result relies on the classification of finite simple groups.) By inspection of this list, we see that the only such doubly transitive groups of degree $p^{2}$ are as follows.

Theorem 6 Let $G$ be a doubly transitive group of degree $p^{2}$ with nonabelian socle. Then either $G=A_{p^{2}}$, or $G=S_{p^{2}}$, or $\operatorname{PSL}(d, q) \leq G \leq \operatorname{P\Gamma L}(d, q)$. 


\subsection{Imprimitive groups with elementary abelian Sylow p-subgroup}

In [22, Proposition B], Jones determined the imprimitive permutation groups of degree $p^{2}$ whose Sylow $p$-subgroup is elementary abelian of order $p^{2}$.

Theorem 7 (Jones, [22]) Let $G$ be an imprimitive permutation group of degree $p^{2}$, where $p$ is prime, such that a Sylow $p$-subgroup of $H$ is elementary abelian of order $p^{2}$. Then $G \leq S_{p} \times S_{p}$

The following simple lemma expresses the conclusion of Theorem 7 more concretely.

Lemma 1 Let $G$ be a transitive subgroup of $S_{p^{2}}$. The following are equivalent:

1. $G \leq S_{p} \times S_{p}$.

2. There are transitive subgroups $H$ and $K$ of $S_{p}$, such that $H \times K \leq G \leq N_{S_{p}}(H) \times$ $N_{S_{p}}(K)$.

3. There are transitive subgroups $H$ and $K$ of $S_{p}$, and a homomorphism $f: H \rightarrow N_{S_{p}}(K) / K$, such that $G=\left\{(\sigma, \tau) \in H \times N_{S_{p}}(K): f(\sigma)=\tau K\right\}$.

Proof: $(1 \Rightarrow 2)$ Let $H=G \cap\left(S_{p} \times 1\right)$ and $K=G \cap\left(1 \times S_{p}\right)$, so $H, K \triangleleft G$. Then $G \leq N_{S_{p} \times S_{p}}(H \times K)=N_{S_{p}}(H) \times N_{S_{p}}(K)$.

$(1 \Rightarrow 3)$ Let $H$ be the image of $G$ under the projection to the first factor, and let $K=G \cap$ $\left(1 \times S_{p}\right)$. Then $G \leq H \times N_{S_{p}}(K)$. By definition of $K$, we have $(G / K) \cap\left[1 \times\left(N_{S_{p}}(K) / K\right)\right]=$ 1 , so $G / K$ is the graph of a well-defined homomorphism $f: H \rightarrow N_{S_{p}}(K) / K$. The desired conclusion follows.

$(2 \Rightarrow 1)$ and $(3 \Rightarrow 1)$ are obvious.

\subsection{Simply primitive groups}

The simply primitive groups of degree $p^{2}$ are given by the following theorem of Wielandt [34, Theorems 8.5 and 16.2]. (Recall that any subgroup $H$ as in part (2) of this result is described in Lemma 1.)

Theorem 8 (Wielandt, [34]) Let $G$ be a simply primitive permutation group of degree $p^{2}$, where $p$ is prime. Then the Sylow p-subgroups of $G$ are elementary abelian of order $p^{2}$, and either

1. G has a unique elementary abelian Sylow p-subgroup, or

2. $G$ has an imprimitive subgroup $H$ of index 2 (and, from Theorem 7 , we have $H \leq$ $\left.S_{p} \times S_{p}\right)$.

\section{The extension of Burnside's Theorem}

In view of the results in Section 2, this section is mainly concerned with imprimitive groups $G$ of degree $p^{2}$ whose Sylow $p$-subgroups $P$ are not elementary abelian. We begin in a slightly more general context. 
Let $G$ be a transitive permutation group of degree $m p$ acting on $\mathbb{Z}_{m} \times \mathbb{Z}_{p}$ that admits a complete block system $\mathcal{B}$ of $m$ blocks of cardinality $p$. We may suppose without loss of generality that $G$ acts on $\mathbb{Z}_{m} \times \mathbb{Z}_{p}$ such that $\mathcal{B}=\left\{\{i\} \times \mathbb{Z}_{p}: i \in \mathbb{Z}_{p}\right\}$. If $g \in G$, then $g$ permutes the $m$ blocks of $\mathcal{B}$ and hence induces a permutation in $S_{m}$ denoted $g / \mathcal{B}$. We define $G / \mathcal{B}=\{g / \mathcal{B}: g \in G\}$. Let $\operatorname{fix}_{G}(\mathcal{B})=\{g \in G: g(B)=B$ for every $B \in \mathcal{B}\}$. Assume that $\operatorname{fix}_{G}(\mathcal{B}) \neq 1$ so that a Sylow $p$-subgroup $P_{0}$ of $\operatorname{fix}_{G}(\mathcal{B})$ is nontrivial. Then $P_{0}$ is contained in $\left\langle z_{i}: i \in \mathbb{Z}_{m}\right\rangle$, where each $z_{i}$ is a $p$-cycle that permutes the elements of $\{i\} \times \mathbb{Z}_{p}$. For $h \in P_{0}$, we then have that $h=\prod_{i=0}^{m-1} z_{i}^{a_{i}}, a_{i} \in \mathbb{Z}_{p}$. Define $v: P_{0} \rightarrow Z_{p}^{m}$ by $v(h)=\left(a_{0}, a_{1}, \ldots, a_{m-1}\right)$.

Lemma 2 The set $\left\{v(h): h \in P_{0}\right\}$ is a linear code of length $m$ over $\mathbb{F}_{p}$.

Proof: As a linear code of length $m$ over $\mathbb{F}_{p}$ is simply an $m$-dimensional vector space over $\mathbb{F}_{p}$, we need only show that $\left\{v(h): h \in P_{0}\right\}$ is a vector space. Note that for $g, h \in P_{0}$ and $r \in \mathbb{Z}_{p}$, we have

$$
g^{r}=\left(\prod_{i=0}^{m-1} z_{i}^{a_{i}}\right)^{r}=\prod_{i=0}^{m-1} z_{i}^{r a_{i}}
$$

and

$$
g h=\prod_{i=0}^{m-1} z_{i}^{a_{i}} \prod_{i=0}^{m-1} z_{i}^{b_{i}}=\prod_{i=0}^{m-1} z_{i}^{a_{i}+b_{i}} .
$$

Hence $v\left(g^{r}\right)=r v(g)$ and $v(g h)=v(g)+v(h)$, so $\left\{v(h): h \in P_{0}\right\}$ is a linear code.

Definition 4 The code of Lemma 2 will be denoted by $C_{\mathcal{B}}$, and will be called the code induced by $\mathcal{B}$. If $G$ admits a unique block system $\mathcal{B}$ of $m$ blocks of cardinality $p$, we say $C_{\mathcal{B}}$ is the code over $\mathbb{F}_{p}$ induced by $G$. We remark that $C_{\mathcal{B}}$ depends upon the choice of the Sylow $p$-subgroup $P_{0}$, but that different choices of $P_{0}$ give monomially equivalent (that is, isomorphic) codes.

Remark Lemma 2 was proven in a less general context in [17].

Lemma 3 If there exists $x \in G$ such that $x(i, j)=\left(i+1, \alpha j+b_{i}\right), b_{i} \in \mathbb{Z}_{p}, \alpha \in \mathbb{F}_{p}^{*}$, then $\left\{v(h): h \in P_{0}\right\}$ is a cyclic code of length $m$ over $\mathbb{F}_{p}$. Conversely, if $C$ is a cyclic code of length $m$ over $\mathbb{F}_{p}$, then there exists a group $G$ as above such that $P_{0}=\left\{\prod_{i=0}^{m-1} z_{i}^{a_{i}}\right.$ : $\left.\left(a_{0}, a_{1}, \ldots, a_{m-1}\right) \in C\right\}$.

Proof: From the form of $x$, we know that $x$ normalizes $\left\langle z_{i}: i \in \mathbb{Z}_{m}\right\rangle$. Also, because $x \in G$, we know that $x$ normalizes fix ${ }_{G}(\mathcal{B})$. Thus, $x$ normalizes $\left\langle z_{i}: i \in \mathbb{Z}_{m}\right\rangle \cap \operatorname{fix}_{G}(\mathcal{B})=P_{0}$.

For $h=\prod z_{i}^{a_{i}} \in P_{0}$ we have $x^{-1} h x=\prod z_{i}^{\alpha a_{i+1}}$, so, because $x$ normalizes $P_{0}$, we see that the linear code $\left\{v(h): h \in P_{0}\right\}$ is cyclic.

Conversely, define $x: \mathbb{Z}_{m} \times \mathbb{Z}_{p} \rightarrow \mathbb{Z}_{m} \times \mathbb{Z}_{p}$ by $x(i, j)=(i+1, j)$. Then it is also straightforward to check that $G=\left\{x^{i} g: i \in \mathbb{Z}_{m}, g \in C\right\}$ will do. 
In the following we consider the case $m=p$, where $\mathbb{Z}_{p} \times \mathbb{Z}_{p}$ is identified with $\mathbb{Z}_{p^{2}}$ via $(a, b) \mapsto a+b p$.

Definition 5 Let $a_{i, j}=\left(\begin{array}{c}i \\ j\end{array}\right)(-1)^{i-j}$. A straightforward calculation will show that $a_{i, j-1}=$ $a_{i+1, j}+a_{i, j}$. For $1 \leq i \leq p$, let

$$
\gamma_{i}=z_{0}^{a_{p-i, 0}} z_{1}^{a_{p-i, 1}} \ldots z_{p-1}^{a_{p-i, p-1}}
$$

Define $\tau: \mathbb{Z}_{p^{2}} \rightarrow \mathbb{Z}_{p^{2}}$ by

$$
\tau(i)=i+1\left(\bmod p^{2}\right)
$$

and $\rho_{1}, \rho_{2}: \mathbb{Z}_{p}^{2} \rightarrow \mathbb{Z}_{p}^{2}$ by

$$
\rho_{1}(i, j)=(i, j+1) \quad \text { and } \quad \rho_{2}(i, j)=(i+1, j) .
$$

Using the above identification of $\mathbb{Z}_{p} \times \mathbb{Z}_{p}$ with $\mathbb{Z}_{p^{2}}$, we have, for example,

$$
\begin{gathered}
z_{i}(a+b p)= \begin{cases}a+b p & \text { if } a \neq i \\
a+(b+1) p\left(\bmod p^{2}\right) & \text { if } a=i,\end{cases} \\
\rho_{1}=\prod_{i=1}^{p-1} z_{i} \text { and } \rho_{2}(a+b p)=(a+1)(\bmod p)+b p . \text { Hence } \tau=z_{p-1} \rho_{2} . \text { Let } \\
P_{i}=\left\langle\tau, \gamma_{i}\right\rangle \quad \text { and } \quad P_{i}^{\prime}=\left\langle\rho_{1}, \rho_{2}, \gamma_{i}\right\rangle,
\end{gathered}
$$

for $1 \leq i \leq p$. We remark that $P_{p}=P_{p}^{\prime} \cong \mathbb{Z}_{p} 2 \mathbb{Z}_{p}$. There are thus $2 p-1$ distinct groups $P_{i}, P_{i}^{\prime}, 1 \leq i \leq p$.

Theorem 9 Let $G$ be a transitive group of degree $p^{2}$ with Sylow $p$-subgroup P. Let $|P|=p^{i+1}, i \geq 1$.

- If $\tau \in P$, then $P=P_{i}$.

- If $\left\langle\rho_{1}, \rho_{2}\right\rangle \leq P$, then $P=\alpha^{-1} P_{i}^{\prime} \alpha$ for some $\alpha \in \operatorname{Aut}\left(\mathbb{Z}_{p}^{2}\right)$.

Proof: By [27], if $C$ is a cyclic code of length $p$ over $\mathbb{F}_{p}$, then $C$ has generator polynomial $f(x)$, where $f(x)$ divides $x^{p}-1$ in $\mathbb{Z}_{p}[x]$. By the Freshman's Dream [21], $x^{p}-1=(x-1)^{p}$ so that $f(x)=(x-1)^{i}$ for some $0 \leq i \leq p-1$. As $C$ is generated by the cyclic shifts of the vector $\left(a_{i, 0}, a_{i, 1}, \ldots, a_{i, p-1}\right)$ where $(x-1)^{i}=\sum_{j=0}^{i} a_{i, j} x^{j}$, we have $a_{i, j}=\left(\begin{array}{c}i \\ j\end{array}\right)(-1)^{i-j}$. Finally, we remark that the dimension of the code $C$ is $p-i$.

Let $G$ be a transitive group of degree $p^{2}$ such that $\tau \in G$. Let $P$ be the Sylow $p$-subgroup of $G$ that contains $\tau$. Then $P$ admits a complete block system $\mathcal{B}$ of $p$ blocks of cardinality $p$ formed by the orbits of $\left\langle\tau^{p}\right\rangle$. Then $\left|\mathrm{fix}_{G} \mathcal{B}\right|=p^{i}$ and $C=\left\{v(g): g \in \mathrm{fix}_{G}(\mathcal{B})\right\}$ is a cyclic code of length $p$ over $\mathbb{F}_{p}$ by Lemma 3 , so that $C$ contains $p^{i}$ codewords and is thus of dimension $i$. We conclude that $P=\left\langle\tau, \prod_{i=0}^{p-1} z_{i}^{a_{i}}:\left(a_{0}, a_{1}, \ldots, a_{p-1}\right) \in C\right\rangle=\left\langle\tau, \gamma_{i}\right\rangle$.

If $\left\langle\rho_{1}, \rho_{2}\right\rangle \leq P$, then again $P$ admits a complete block system $\mathcal{B}$ formed by the orbits of $\langle\delta\rangle$, where $\langle\delta\rangle=\left\langle\rho_{1}\right\rangle$ or $\left\langle\rho_{1}^{i} \rho_{2}\right\rangle, 0 \leq i \leq p-1$. Hence there exists a group 
automorphism $\alpha$ of $\mathbb{Z}_{p}^{2}$ such that $\alpha^{-1} \delta \alpha=\rho_{2}$. It then follows by arguments above that $P=\alpha^{-1}\left\langle\rho_{1}, \rho_{2}, \gamma_{i}\right\rangle \alpha$.

Remark Every transitive group $G$ of degree $p^{2}$ contains a subgroup isomorphic to either $\langle\tau\rangle$ or $\left\langle\rho_{1}, \rho_{2}\right\rangle$, as every Sylow $p$-subgroup of $G$ is transitive and contains a nontrivial center. Hence the above result determines all transitive $p$-subgroups of $S_{p^{2}}$ up to isomorphism.

Remark Theorem 9 was already proven in [19] for the case where $P$ contains a regular subgroup isomorphic to $\langle\tau\rangle$.

Lemma 4 Let $P$ be a transitive $p$-subgroup of $S_{p^{2}}$. Then $P$ admits a complete block system $\mathcal{B}$ of $p$ blocks of cardinality $p$.

Furthermore, the following are equivalent:

1. $P$ does not contain regular copies of both $\mathbb{Z}_{p^{2}}$ and $\mathbb{Z}_{p}^{2}$.

2. $P ¥ \mathbb{Z}_{p}>\mathbb{Z}_{p}$.

3. Letting $C$ be the code induced by $\mathcal{B}$, we have $\sum_{i=0}^{p-1} a_{i} \equiv 0(\bmod p)$, for every $\left(a_{0}, a_{1}, \ldots, a_{p-1}\right) \in C$.

Proof: $\quad(1 \Rightarrow 2)$ If $P \cong \mathbb{Z}_{p} 2 \mathbb{Z}_{p}$, then $P$ is a Sylow $p$-subgroup of $S_{p^{2}}$, so it is clear that $P$ contains both a regular subgroup isomorphic to $\mathbb{Z}_{p^{2}}$ and a regular subgroup isomorphic to $\mathbb{Z}_{p}^{2}$.

$(2 \Rightarrow 1)$ Assume $P$ contains regular copies of both $\mathbb{Z}_{p^{2}}$ and $\mathbb{Z}_{p}^{2}$. Without loss of generality assume that $\tau \in P$. As $P$ contains a nontrivial center, $\tau^{p} \in Z(P)$, so that $P$ admits a complete block system $\mathcal{B}$ of $p$ blocks of cardinality $p$ formed by the orbits of $\left\langle\tau^{p}\right\rangle$. As $P$ contains a regular subgroup isomorphic to $\mathbb{Z}_{p}^{2}$, there exists $\tau_{1}, \tau_{2} \in P$ such that $\left\langle\tau_{1}, \tau_{2}\right\rangle \cong \mathbb{Z}_{p}^{2}$. As $|P / \mathcal{B}|=p$, we assume without loss of generality that $\tau_{2} / \mathcal{B}=1$ so that $\left|\tau_{1} / \mathcal{B}\right|=p$. As $\left|\tau_{1}\right|=p, \tau_{1}^{-1} \tau^{p} \tau_{1}=\tau^{p}$ and $\left|\tau^{p}\right|=p$, we may assume that $\tau_{2}=\tau^{p}$. We regard $\mathbb{Z}_{p^{2}}$ as $\mathbb{Z}_{p}^{2}$. Hence $\tau(i, j)=\left(i+1, \delta_{i}(j)\right)$ where $\delta_{i}(j)=j, 0 \leq i \leq p-2$ and $\delta_{p-1}(j)=j+1$. Further, $\tau_{1}(i, j)=\left(i+r, j+b_{i}\right), r, b_{i} \in \mathbb{Z}_{p}$. As $\left|\tau_{1}\right|=p, \sum_{i=0}^{p-1} b_{i} \equiv 0(\bmod p)$. We assume without loss of generality that $r=1$. Then $\tau^{-1} \tau_{1}(i, j)=\left(i, j+c_{i}\right)$ where $\sum_{i=0}^{p-1} c_{i} \equiv-1(\bmod p)$. Then $\operatorname{fix}_{P}(\mathcal{B})=\left\langle\tau, \tau^{-j} \gamma_{i} \tau^{j}: 1 \leq j \leq p-1\right\rangle$, for some $1 \leq i \leq p$. If $1 \leq k \leq p-1$ and $\psi \in P_{k}$ with $\psi(i, j)=\left(i, j+d_{i}\right)$, we have that $\sum_{i=0}^{p-1} d_{i} \equiv 0(\bmod p)$. Hence $i=p$ and $\left\langle\tau, \tau^{-1} \tau_{1}\right\rangle \cong \mathbb{Z}_{p} 2 \mathbb{Z}_{p}$ as required.

(3 $\Rightarrow$ ) Obvious.

( $2 \Rightarrow 3$ ) If $P ¥ \mathbb{Z}_{p} 2 \mathbb{Z}_{p}$, then, from the proof of Theorem 9 , we see that the generating polynomial of $C$ is divisible by $x-1$. The desired conclusion follows.

We now calculate the normalizers of $P_{i}$ and $P_{i}^{\prime}, i \leq p$. (We remark that the normalizer of each $P_{i}$ was calculated in [19].)

Definition 6 For $\beta \in \mathbb{F}_{p}^{*}$, define $\bar{\beta}, \tilde{\beta}: \mathbb{Z}_{p}^{2} \rightarrow \mathbb{Z}_{p}^{2}$ by

$$
\bar{\beta}(i, j)=(\beta i, j) \quad \text { and } \quad \tilde{\beta}(i, j)=(i, \beta j)
$$


For $\beta \in \mathbb{Z}_{p^{2}}^{*}$ define $\hat{\beta}: \mathbb{Z}_{p^{2}} \rightarrow \mathbb{Z}_{p^{2}}$ by

$$
\hat{\beta}(i)=\beta i .
$$

Remark Of course, $N_{S_{p^{2}}}\left(P_{i}\right)$ admits a (unique) complete block system $\mathcal{B}$ of $p$ blocks of cardinality $p$ formed by the orbits of $\left\langle\tau^{p}\right\rangle$. It is straightforward to show that $\operatorname{fix}_{N_{S_{p^{2}}}\left(P_{i}\right)}(\mathcal{B})$ is a $p$-group of order $p^{i}, 1 \leq i \leq p-1$.

Lemma 5 ([19]) We have

$$
N_{S_{p^{2}}}\left(P_{i}\right)= \begin{cases}P_{i+1} \rtimes\left\{\hat{\beta}: \beta \in \mathbb{Z}_{p^{2}}^{*},|\beta| \in\{1, p-1\}\right\} & \text { if } 1 \leq i \leq p-1 \\ P_{p} \rtimes\left\{\bar{\beta}, \tilde{\beta}: \beta \in \mathbb{F}_{p}^{*}\right\} & \text { if } i=p .\end{cases}
$$

Proof: It is well known that

$$
N_{S_{p^{2}}}\left(P_{1}\right)=N_{S_{p^{2}}}(\langle\tau\rangle)=\left\{x \mapsto a x+b: a \in \mathbb{Z}_{p^{2}}^{*}, b \in \mathbb{Z}_{p^{2}}\right\}
$$

and it essentially follows by arguments in [1] and was explicitly shown in [19] that

$$
N_{S_{p^{2}}}\left(P_{p}\right)=P_{p} \rtimes\left\{\bar{\beta}, \tilde{\beta}: \beta \in \mathbb{F}_{p}^{*}\right\},
$$

so we may assume $2 \leq i \leq p-1$.

We first show that $\left|N_{S_{p^{2}}}\left(P_{i}\right)\right|=(p-1) p^{i+2}$.

Let $X=\left\{\langle x\rangle:\langle x\rangle\right.$ is a regular cyclic subgroup of $\left.S_{p^{2}}\right\}$ and let $S_{p^{2}}$ act on $X$ by conjugation. Denote the resulting transitive permutation group on $X$ by $\Delta$. Note that there are $p^{2} ! /(p-1) p^{3}=\left[S_{p^{2}}: N_{S_{p^{2}}}(\langle\tau\rangle)\right]$ elements of $X$. As $\left\langle\tau^{p}\right\rangle \leq Z\left(P_{i}\right)$ and is the unique subgroup of $Z\left(P_{i}\right)$ of order $p, N_{S_{p^{2}}}\left(P_{i}\right)$ admits a complete block system $\mathcal{B}$ of $p$ blocks of cardinality $p$, formed by the orbits of $\left\langle\tau^{p}\right\rangle$. Observe that if $\langle x\rangle \in X$ and $\langle x\rangle \leq P_{i}$, then we may assume that $x=\tau \gamma, \gamma \in \operatorname{fix}_{P_{i}}(\mathcal{B})$. Then $\left|\operatorname{fix}_{P_{i}}(\mathcal{B})\right|=p^{i}$, and by Lemma $4 \tau \gamma$ is a $p^{2}$-cycle for every $\gamma \in \operatorname{fix}_{P_{i}}(\mathcal{B})$ as every minimal transitive subgroup of $P_{i}$ is isomorphic to $\mathbb{Z}_{p^{2}}$. Furthermore, there are exactly $p$ elements of $\langle\tau \gamma\rangle$ contained in $\operatorname{fix}_{P_{i}}(\mathcal{B})$. We conclude that $P_{i}$ contains $p^{i} / p=p^{i-1}$ elements of $X$. Let $B=\left\{\langle\tau \gamma\rangle: \gamma \in\right.$ fix $\left._{P_{i}}(\mathcal{B})\right\}$. We first will show that $B$ is a block of $\Delta$.

Let $\delta \in S_{p^{2}}$ such that $\delta^{-1} B \delta \cap B \neq \emptyset$. Then there exists $x=\tau \gamma, \gamma \in \operatorname{fix}_{P_{i}}(\mathcal{B})$ such that $\delta^{-1}\langle x\rangle \delta \leq P_{i}$. Then $\delta^{-1}\langle x\rangle \delta / \mathcal{B}=\langle x\rangle / \mathcal{B}$ and hence $\delta^{-1}\langle y\rangle \delta / \mathcal{B}=\langle\tau\rangle / \mathcal{B}$ for every $\langle y\rangle \in B$. Then $\left\langle\delta^{-1} B \delta\right\rangle$ satisfies the hypothesis of Lemma 3 (as $\delta^{-1}\langle x\rangle \delta \leq P_{i}$ ) so the code corresponding to $\left\langle\delta^{-1} B \delta\right\rangle$ is the code corresponding to $\langle B\rangle$ which implies $\left\langle\delta^{-1} B \delta\right\rangle=\langle B\rangle$ so that $\delta^{-1} B \delta=B$ as required. Hence the number of subgroups conjugate in $S_{p^{2}}$ to $P_{i}=\langle B\rangle$ is the number of blocks conjugate to $B$ in $\Delta$. As there are $\left(p^{2} ! / p^{3}(p-1)\right) / p^{i-1}$ such blocks, $\left|N_{S_{p^{2}}}\left(P_{i}\right)\right|=(p-1) p^{i+2}$ as required.

It is straightforward to check using the recursion formula given in Definition 5 that $\gamma_{i+1} \in N_{S_{p^{2}}}\left(P_{i}\right)$. Note that the result is clearly true for $i=1$ as $\left\langle\tau, \gamma_{2}\right\rangle \leq N_{S_{p^{2}}}(\langle\tau\rangle)$. Hence $N_{S_{p^{2}}}(\langle\tau\rangle) \leq N_{S_{p^{2}}}\left(\left\langle\tau, \gamma_{3}\right\rangle\right)$ as $\left\langle\tau, \gamma_{2}\right\rangle$ is the unique Sylow $p$-subgroup of $N_{S_{p^{2}}}(\langle\tau\rangle)$. Continuing inductively, we have that $N_{S_{p^{2}}}(\langle\tau\rangle) \leq N_{S_{p^{2}}}\left(\left\langle\tau, \gamma_{i}\right)\right.$ and as $\left|\left\langle N_{S_{p^{2}}}(\langle\tau\rangle), \gamma_{i}\right\rangle\right|=$ $(p-1) p^{i+2}$, the result follows. 
Lemma 6 We have

$$
N_{S_{p^{2}}}\left(P_{i}^{\prime}\right)= \begin{cases}\operatorname{AGL}(2, p) & \text { if } i=1 \\ P_{i+1}^{\prime} \rtimes\left\{\bar{\beta}, \tilde{\beta}: \beta \in \mathbb{F}_{p}^{*}\right\} & \text { if } 2 \leq i \leq p-1 \\ P_{p}^{\prime} \rtimes\left\{\bar{\beta}, \tilde{\beta}: \beta \in \mathbb{F}_{p}^{*}\right\} & \text { if } i=p .\end{cases}
$$

Proof: The case $i=1$ is well known, and the case $i=p$ appears in Lemma 5, so we may assume $2 \leq i \leq p-1$.

Because $i \geq 2$, we know that $\left\langle\tau_{2}\right\rangle=Z\left(P_{i}^{\prime}\right)$ is characteristic in $P_{i}^{\prime}$, so that $\left\langle\tau_{2}\right\rangle \triangleleft N_{S_{p^{2}}}\left(P_{i}^{\prime}\right)$. Hence if $\delta \in N_{S_{p^{2}}}\left(P_{i}^{\prime}\right)$, then $\delta(i, j)=\left(\sigma(i), \gamma j+b_{i}\right), \sigma \in S_{p}, \gamma \in \mathbb{F}_{p}^{*}, b_{i} \in \mathbb{Z}_{p}$. Furthermore, $\sigma(i)=\beta i+b, \beta \in \mathbb{F}_{p}^{*}, b \in \mathbb{Z}_{p}$. It is straightforward to check that $\bar{\beta}, \tilde{\gamma} \in N_{S_{p^{2}}}\left(P_{i}^{\prime}\right)$, so we may assume $\beta=\gamma=1$. As $\tau_{1} \in P_{i}^{\prime}$, we may assume without loss of generality that $b=0$. As $\gamma_{1}, \gamma_{2}, \ldots, \gamma_{i} \in P_{i}^{\prime}$, we may assume, for $0 \leq k \leq i-1$, that $b_{k}=0$. It is then straightforward to show that $\delta \in\left\langle\gamma_{i+1}\right\rangle$.

Definition 7 A code $C$ of length $m$ over $\mathbb{F}_{p}$ is said to be degenerate if there exists $k \mid m, k \neq m$, and a code $D$ of length $k$ over $\mathbb{F}_{p}$ such that $C=\bigoplus_{i=1}^{m / k} D$. That is, a code $C=\left\{\left(d_{1}, d_{2}, \ldots, d_{m / k}\right) \mid d_{1}, \ldots, d_{m / k} \in D\right\}$. If $C$ is not degenerate, we say that it is nondegenerate.

Lemma 7 Let $G \leq S_{p^{2}}$ admit a complete block system $\mathcal{B}$ of p blocks of cardinality $p$. If fix $_{G}(\mathcal{B})$ contains at least two Sylow p-subgroups and $C_{\mathcal{B}}$ is nondegenerate, then a Sylow p-subgroup of $G$ is isomorphic to $\mathbb{Z}_{p} \times \mathbb{Z}_{p}$.

Proof: We assume that $\langle\tau\rangle \leq G$ or $\left\langle\rho_{1}, \rho_{2}\right\rangle \leq G$. Let $P$ be a Sylow $p$-subgroup of $G$ that contains $\langle\tau\rangle$ or $\left\langle\rho_{1}, \rho_{2}\right\rangle$. Observe that $P$ cannot contain both $\langle\tau\rangle$ and $\left\langle\rho_{1}, \rho_{2}\right\rangle$, for then Lemma 4 would imply $P \cong \mathbb{Z}_{p} \imath \mathbb{Z}_{p}$, in which case $C_{\mathcal{B}}$ is degenerate. If fix $\operatorname{fix}_{G}(\mathcal{B})$ contains at least two Sylow $p$-subgroups, then $\left.\operatorname{fix}_{G}(\mathcal{B})\right|_{B}$ contains at least two Sylow $p$-subgroups for every $B \in \mathcal{B}$ and hence, by the comments following Theorem 1, is nonsolvable. By Theorem $\left.1 \operatorname{fix}_{G}(\mathcal{B})\right|_{B}$ is doubly transitive for every $B \in \mathcal{B}$.

Suppose, for the moment, that $\operatorname{fix}_{P}(\mathcal{B})$ is faithful on some block of $\mathcal{B}$. Then a Sylow $p$-subgroup of $G$ has order $p^{2}$. If $P=\left\langle\rho_{1}, \rho_{2}\right\rangle$, we are finished, so we assume that $P=$ $\langle\tau\rangle$ and hence $\operatorname{fix}_{P}(\mathcal{B})=\left\langle\tau^{p}\right\rangle$. Clearly $\left.\left\langle\tau^{p}\right\rangle\right|_{B}$ is a Sylow $p$-subgroup of $\left.\operatorname{fix}_{G}(\mathcal{B})\right|_{B}$, and if $N_{\left.\mathrm{fix}_{G}(\mathcal{B})\right|_{B}}\left(\left.\left\langle\tau^{p}\right\rangle\right|_{B}\right)=\left.\left\langle\tau^{p}\right\rangle\right|_{B}$, then by Burnside's Transfer Theorem [14, Theorem 4.3, p. 252], $\left.\left\langle\tau^{p}\right\rangle\right|_{B}$ has a normal $p$-complement in $\left.\operatorname{fix}_{G}(\mathcal{B})\right|_{B}$. Whence fix $\left.\cos _{G}(\mathcal{B})\right|_{B}$ admits a complete block system of $p$ blocks of cardinality $m$, where $m \neq 1$, a contradiction. Thus $N_{\text {fix }\left._{G}(\mathcal{B})\right|_{B}}\left(\left.\left\langle\tau^{p}\right\rangle\right|_{B}\right) \neq\left.\left\langle\tau^{p}\right\rangle\right|_{B}$, so there exists $\delta \in$ fix $_{G}(\mathcal{B})-\left\langle\tau^{p}\right\rangle$ such that $\delta^{-1} \tau^{p} \delta=\tau^{a p}$, $a \neq 1$. By the remark preceding Lemma $5, \delta \notin N_{S_{p^{2}}}(\langle\tau\rangle)$, so that $\delta^{-1} \tau \delta \tau^{-1} \in \operatorname{fix}_{G}(\mathcal{B})$, but $\delta^{-1} \tau \delta \tau^{-1} \notin\left\langle\tau^{p}\right\rangle$. A straightforward computation will then show that $\delta^{-1} \tau \delta \tau^{-1}$ centralizes $\left\langle\tau^{p}\right\rangle$. As $\delta^{-1} \tau \delta \tau^{-1} \in \operatorname{fix}_{G}(\mathcal{B}),\left.\delta^{-1} \tau \delta \tau^{-1}\right|_{B}$ centralizes $\left.\left\langle\tau^{p}\right\rangle\right|_{B}$, and of course, $\left.\left\langle\tau^{p}\right\rangle\right|_{B}$ is regular and abelian. As a regular abelian group is self-centralizing [33, Proposition 4.4], we conclude that $\left.\left.\delta^{-1} \tau \delta \tau^{-1}\right|_{B} \in\left\langle\tau^{p}\right\rangle\right|_{B}$. Whence $\delta^{-1} \tau \delta \tau^{-1}$ has order $p$ and $\left\langle\tau^{p}, \delta^{-1} \tau \delta \tau^{-1}\right\rangle \leq$ $\operatorname{fix}_{G}(\mathcal{B})$ and has order $p^{2}$, a contradiction. 
Henceforth, we assume that $\operatorname{fix}_{P}(\mathcal{B})$ is not faithful on any block of $\mathcal{B}$, so the Sylow $p$ subgroups of $\operatorname{fix}_{G}(\mathcal{B})$ have order at least $p^{2}$. Let $\gamma \in \operatorname{fix}_{G}(\mathcal{B})$ such that $\left.\gamma\right|_{B} \neq 1$ for the fewest number of blocks $B \in \mathcal{B}$, and let $\mathcal{C}=\left\{B \in \mathcal{B}:\left.\gamma\right|_{B} \neq 1\right\}$. If $\cup \mathcal{C}$ is a block of $G$, then $C_{\mathcal{B}}$ is degenerate and we are finished. Otherwise, define $\pi: f_{i x}(\mathcal{B}) \rightarrow S_{\cup(\mathcal{B}-\mathcal{C})}$ by $\pi(g)=\left.g\right|_{\cup(\mathcal{B}-\mathcal{C})}$. Then $\gamma \in \operatorname{Ker}(\pi)$. As $\operatorname{Ker}(\pi) \triangleleft \operatorname{fix}_{G}(\mathcal{B})$ and $\left.\operatorname{fix}_{G}(\mathcal{B})\right|_{B}$ is primitive for every $B \in \mathcal{B},\left.\operatorname{Ker}(\pi)\right|_{B}$ is transitive for every $B \in \mathcal{C}$. Hence we may assume $|\gamma|=p$. As any two Sylow $p$-subgroups of $\operatorname{fix}_{G}(\mathcal{B})$ are conjugate and one Sylow $p$-subgroup of $\operatorname{fix}_{G}(\mathcal{B})$ is contained in $\left\langle z_{i}: i \in \mathbb{Z}_{p}\right\rangle$, we assume without loss of generality that $\gamma \in\left\langle z_{i}: i \in \mathbb{Z}_{p}\right\rangle$. Finally, observe that as $\gamma \in \operatorname{fix}_{G}(\mathcal{B})$ such that $\left.\gamma\right|_{B} \neq 1$ for the fewest number of blocks of $\mathcal{B},\langle\gamma\rangle$ is a Sylow $p$-subgroup of $\operatorname{Ker}(\pi)$. As $\operatorname{Ker}(\pi) \triangleleft \operatorname{fix}_{G}(\mathcal{B}),\left.\operatorname{Ker}(\pi)\right|_{B}$ contains at least 2 Sylow $p$-subgroups. It then follows by Burnside's Transfer Theorem that $N_{\operatorname{Ker}(\pi)}(\langle\gamma\rangle) \neq$ $\langle\gamma\rangle$.

Let $\gamma=z_{i_{1}}^{a_{i_{1}}} z_{i_{2}}^{a_{i_{2}}} \ldots z_{i_{r}}^{a_{i_{r}}}$. Let $\delta \in N_{\operatorname{Ker}(\pi)}(\langle\gamma\rangle)$ such that $\delta \notin\langle\gamma\rangle$. Then $\delta^{-1} \gamma \delta=z_{i_{1}}^{b a_{i_{1}}}$ $z_{i_{2}}^{b a_{i_{2}}} \ldots z_{i_{r}}^{b a_{i_{r}}}$, for some $b \in \mathbb{Z}_{p}^{*}$. As $\cup \mathcal{C}$ is not a block of $G$, there exists $\iota \in G$ such that $\iota^{-1}(\cup \mathcal{C}) \iota \cap(\cup \mathcal{C}) \neq \emptyset$ and $\iota^{-1}(\cup \mathcal{C}) \iota \neq \cup \mathcal{C}$. Let $\iota^{-1} \gamma \iota=z_{j_{1}}^{c_{j_{1}}} z_{j_{2}}^{c_{j_{2}}} \ldots z_{j_{r}}^{c_{j_{r}}}$, for some $j_{1}, j_{2}, \ldots$, $j_{r} \in \mathbb{Z}_{p^{k-1}}$ and $c_{j_{\ell}} \in \mathbb{Z}_{p}^{*}$. Then $\left.\delta^{-1} \iota^{-1} \gamma \iota \delta\left(\iota^{-1} \gamma \iota\right)^{-b}\right|_{B} \neq 1$ for fewer blocks of $\mathcal{B}$ than $\gamma$, a contradiction.

Definition 8 For a code $C$ of length $n$ over a field $\mathbb{F}_{p}$ of prime order $p$, let $\operatorname{Aut}(C)$ be the group of all linear bijections of $K^{n}$ which map each codeword of $C$ to a codeword of $C$ of the same weight. Thus $\operatorname{Aut}(C)$ is the subgroup of $M_{n}\left(\mathbb{F}_{p}\right)$ that map each codeword of $C$ to a codeword of $C$, where $M_{n}\left(\mathbb{F}_{p}\right)$ is the set of all $n \times n$ monomial matrices over $\mathbb{F}_{p}$. That is, matrices with exactly one nonzero entry from $\mathbb{F}_{p}$ in each row and column.

Let $\left[m_{i j}\right]=M \in M_{n}\left(\mathbb{F}_{p}\right)$. Then $M=P D$, where $P=\left[p_{i j}\right]$ is the permutation matrix given by $p_{i j}=1$ if $m_{i j} \neq 0$ and $p_{i j}=0$ otherwise and $D=\left[d_{i j}\right]$ is a diagonal matrix with $d_{i i}=m_{i j}$ if $m_{i j} \neq 0$ and $d_{i j}=0$ if $i \neq j$. As the group of all permutation matrices is simply the symmetric group on the coordinates of a vector in $\mathbb{F}_{p}^{n}$, there is thus a canonical isomorphism between $M_{n}\left(\mathbb{F}_{p}\right)$ and $S_{n} \ltimes\left(\mathbb{F}_{p}^{*}\right)^{n}$, with multiplication in $S_{n} \ltimes\left(\mathbb{F}_{p}^{*}\right)^{n}$ given by $(\sigma, a)(\tau, b)=\left(\sigma \tau,\left(\sigma^{-1} b\right) a\right)$ and $S_{n} \ltimes\left(\mathbb{F}_{p}^{*}\right)^{n}$ acts on $\mathbb{F}_{p}^{n}$ by $(\sigma, d)(x)=\sigma(x d)$. We will abuse notation and write that $(\sigma, d)=M \in M_{n}\left(\mathbb{F}_{p}\right)$. If $(\sigma, d) \in \operatorname{Aut}(C)$, then $(\sigma, d)$ is diagonal if and only if $\sigma=1$. Finally, we let $\operatorname{PAut}(C)=\{\sigma:(\sigma, d) \in \operatorname{Aut}(C)\}$.

Theorem 10 ([26], Theorem 1.3) If $C$ is a nontrivial code such that $\operatorname{PAut}(C)$ is primitive, then $C$ is nondegenerate and every diagonal automorphism of $C$ is scalar.

Definition 9 A code $C$ of length $p$ over $\mathbb{F}_{p}$ is affine invariant if $\operatorname{AGL}(1, p) \leq \operatorname{PAut}(C)$.

Let $P \leq S_{p^{2}}$ be a transitive $p$-group. Then $P$ admits a complete block system $\mathcal{B}$ of $p$ blocks of cardinality $p$, formed by the orbits of a semiregular element of order $p$ contained in the center of $P$. By Theorem 9, we may assume that $P=P_{i}$ or $P_{i}^{\prime}$. Note that if $G \leq S_{p^{2}}$ admits $\mathcal{B}$ as a complete block system with Sylow $p$-subgroup $P$, then conjugation by an element of $G$ induces an automorphism of $C_{\mathcal{B}}$. It then follows by Lemmas 5 and 6 that $C_{\mathcal{B}}$ is affine invariant. 
Theorem 11 ([18], Theorem 3.17) Let $C$ be a cyclic code of length $p$ over $\mathbb{F}_{p}$ such that $|C| \neq p, p^{p-1}$ or $p^{p}$. Then $\operatorname{Aut}(C)=\left\{(\sigma, d): \sigma \in \operatorname{AGL}(1, p)\right.$ and $\left.d \in \mathbb{F}_{p}^{*}\right\}$.

Lemma 8 Let $G \leq S_{p^{2}}$ be transitive such that $G$ admits a complete block system $\mathcal{B}$ of $p$ blocks of cardinality p. Furthermore, assume either $P_{1}$ or $P_{p-2}$ is a Sylow p-subgroup of $G$ and $\operatorname{fix}_{G}(\mathcal{B})$ is a p-group. Then $G / \mathcal{B} \leq \operatorname{AGL}(1, p)$.

Proof: We argue by contradiction and thus assume that $G / \mathcal{B}$ has at least 2 Sylow $p$ subgroups and is doubly transitive. As $G / \mathcal{B}$ has at least 2 Sylow $p$-subgroups and a Sylow $p$-subgroup of $G$ is $P_{1}$ or $P_{p-2}$, there exists $g \in G$ such that $|g / \mathcal{B}|=p, g$ is of order a power of $p$, but $g / \mathcal{B} \notin\langle\tau\rangle / \mathcal{B}$. As $g$ is contained in a Sylow $p$-subgroup of $G$ (which is isomorphic to $P_{1}$ or $\left.P_{p-2}\right)$, we have that $\langle g\rangle$ is cyclic and regular of order $p^{2}$. As $G$ admits $\mathcal{B}$ as a complete block system and $\operatorname{fix}_{G}(\mathcal{B})$ is a $p$-group, we have that $g(i+j p)=$ $\sigma(i)+\left(\alpha_{i} j+b_{i}\right) p, \sigma \in S_{p}, \alpha_{i} \in \mathbb{F}^{*}, b_{i} \in \mathbb{Z}_{p}$. It follows by [18, Theorem 3.16(i)] that $\alpha_{i}=\alpha_{j}$ for all $i \in \mathbb{Z}_{p}$. As $|g|=p^{2^{2}}$, we have that $\alpha_{i}=1$ for all $i, j \in \mathbb{Z}_{p}$. As $|g|=p^{2}$, $\sum_{i=0}^{p-1} b_{i} \equiv c \neq 0(\bmod p)$. Then there exists $r \in \mathbb{Z}_{p}$ such that $g \tau^{r} / \mathcal{B}$ has a fixed point, and $g \tau^{r} / \mathcal{B} \neq 1$. Let $d=\left|g \tau^{r} / \mathcal{B}\right|$, and $\mathcal{O}_{1}, \mathcal{O}_{2}, \ldots, \mathcal{O}_{s}$ the orbits of $\left\langle g \tau^{r} / \mathcal{B}\right\rangle$. Let $d_{i}=\left|\mathcal{O}_{i}\right|$. Then $\left(g \tau^{r}\right)^{d} \in \operatorname{fix}_{G}(\mathcal{B})$ so that if $\left(g \tau^{r}\right)^{d}=z_{0}^{b_{0}} z_{1}^{b_{1}} \ldots z_{p-1}^{b_{p-1}}$, then $\sum_{i=0}^{p-1} b_{i} \equiv 0(\bmod p)$. Let $g \tau^{r}\left(i+j p^{k-1}\right)=\delta(i)+\left(j+c_{i}\right) p$. Then $\sum_{i=0}^{p-1} c_{i} \equiv c+r(\bmod p)$. Further,

$$
\begin{aligned}
\sum_{i=0}^{p-1} b_{i} & =\sum_{i=0}^{s}\left(\sum_{j \in \mathcal{O}_{i}} a_{i}\right) \\
& =\sum_{i=0}^{s} d_{i}\left(\frac{d}{d_{i}} \sum_{j \in \mathcal{O}_{i}} c_{i}\right) \\
& =\sum_{i=0}^{p-1} d c_{i} \\
& \equiv d(c+r)(\bmod p) .
\end{aligned}
$$

Note that as a Sylow $p$-subgroup of $G / \mathcal{B}$ is cyclic, $\operatorname{gcd}(d, p)=1$. As $\sum_{i=0}^{p-1} b_{i} \equiv 0(\bmod p)$ and $(d, p)=1$, we have that $c \equiv-r(\bmod p)$. However, there exists $t \in \mathbb{Z}_{p}^{*}, t \neq r$ such that $g \tau^{t} / \mathcal{B}$ has a fixed point, and analogous arguments will show that $c \equiv-t(\bmod p)$, a contradiction.

Let $G$ be a transitive group acting on $\Omega$, and let $G$ act on $\Omega \times \Omega$ by $g(\alpha, \beta)=(g(\alpha), g(\beta))$. Let $\mathcal{O}_{0}, \mathcal{O}_{1}, \ldots, \mathcal{O}_{n}$ be the orbits of $G$ acting on $\Omega \times \Omega$. We call the orbit $\{(\alpha, \alpha): \alpha \in \Omega\}$ the trivial orbit. Assume $\mathcal{O}_{0}$ is the trivial orbit, and define directed graphs $\Gamma_{1}, \ldots, \Gamma_{n}$ by $V\left(\Gamma_{i}\right)=\Omega$ and $E\left(\Gamma_{i}\right)=\mathcal{O}_{i}$ for each $1 \leq i \leq n$. The graphs $\Gamma_{i}$ are orbital digraphs of $G$. Note that $G \leq \operatorname{Aut}\left(\Gamma_{i}\right)$ for all $1 \leq i \leq n$. We define the 2-closure of $G$, denoted $\operatorname{cl}(G)$ to be $\bigcap_{i=1}^{n} \operatorname{Aut}\left(\Gamma_{i}\right)$.

Proof of Theorem 3: Let $G \leq S_{p^{2}}$ be transitive such that $P \not P_{1}^{\prime}, P_{p-1}^{\prime}$, or $\mathbb{Z}_{p}$ 2 $\mathbb{Z}_{p}$. If $\tau \in P$, then, as $\mathbb{Z}_{p^{2}}$ is a Burnside group [33], $G$ is either doubly transitive or imprimitive (this 
may also be obtained from a result of Guralnick [15]), so we may assume $G$ is imprimitive. If $\tau \notin G$, then, as $p^{3}|| G \mid$, we see from $[1,13]$ that any orbital digraph $\Gamma_{i}$ of $G$ is either complete, empty, or a nontrivial wreath product. We conclude that $\tau \in \operatorname{cl}(G)$ so that $G$ is again imprimitive or doubly transitive. We consider the cases $P \cong P_{i}$ and $P \cong P_{i}^{\prime}$ for some $i$ separately.

If $P \cong P_{i}$, then by Lemma 7 fix $_{G}(\mathcal{B})$ contains a unique Sylow $p$-subgroup. If $\operatorname{fix}_{G}(\mathcal{B})$ is a $p$-group and $P \cong P_{1}$ or $P_{p-2}$, then it follows by Lemma 8 that $G / \mathcal{B} \leq A G L(1, p)$. Then $G / \mathcal{B}$ contains exactly one Sylow $p$-subgroup, and $P \triangleleft G$. If $\operatorname{fix}_{G}(\mathcal{B})$ is a $p$-group and $P ¥ P_{1}$ or $P_{p-2}$, then it follows by Theorem 11 that $G / \mathcal{B} \leq A G L(1, p)$ and the result follows. If $\operatorname{fix}_{G}(\mathcal{B})$ is not a $p$-group, then observe that conjugation of the unique Sylow $p$-subgroup of fix $_{G}(\mathcal{B})$ by an element of $G$ induces an automorphism of $C_{\mathcal{B}}$, the code induced by $\mathcal{B}$. Let $g \in \mathrm{fix}_{G}(\mathcal{B})$ such that $g$ is not in the unique Sylow $p$-subgroup of $G$. Let $(\sigma, d)$ be the automorphism of $C_{\mathcal{B}}$ induced by conjugating the unique Sylow $p$-subgroup of $\operatorname{fix}_{G}(\mathcal{B})$ by $g$. Then $\sigma=1$ so that $(\sigma, d)$ is diagonal. As $C_{\mathcal{B}}$ has prime length, $\operatorname{PAut}\left(C_{\mathcal{B}}\right)$ is primitive, and hence by Theorem 10, we have that $(\sigma, d)$ is scalar. Whence $g(i+j p)=i+\left(d j+b_{i}\right) p$. A straightforward computation will then show that $g^{-1} \tau g \tau^{-1}$ is in the unique Sylow $p$-subgroup of fix ${ }_{G}(\mathcal{B})$ so that $g^{-1} \tau g \in P_{i}$. Thus $g \in N_{S_{p^{2}}}\left(P_{i}\right)$. However, by Lemma 5 , $\operatorname{fix}_{N_{S_{p^{2}}}\left(P_{i}\right)}(\mathcal{B})$ contains no element of order relatively prime to $p$, a contradiction.

If $P \cong P_{i}^{\prime}$, then by Lemma $7, \operatorname{fix}_{G}(\mathcal{B})$ contains exactly one Sylow $p$-subgroup, and by arguments in the immediately preceding paragraph, if $g \in \operatorname{fix}_{G}(\mathcal{B})$ such that $|g| \neq$ $p$, then $g(i, j)=\left(i, d^{-1} j+b_{i}\right), d \in \mathbb{F}_{p}^{*}, b_{i} \in \mathbb{Z}_{p}$. It is then straightforward to verify that $g \in N_{S_{p^{2}}}\left(P_{i}^{\prime}\right)$. By Theorem 11, $\left\langle\tau_{1}\right\rangle / \mathcal{B} \triangleleft G / \mathcal{B}$. Thus $H=\left\langle P_{i}^{\prime}, \operatorname{fix}_{G}(\mathcal{B})\right\rangle \triangleleft G$, and $P_{i}^{\prime} \triangleleft H$. As $P_{i}^{\prime}$ is a Sylow $p$-subgroup of $G$, it is the unique Sylow $p$-subgroup of $H$ and so $P_{i}^{\prime}$ is characteristic in $H$. As $H \triangleleft G$, we have that $P_{i}^{\prime} \triangleleft G$.

\section{Overgroups of $\mathbb{Z}_{p}^{2}$ and its "dual"}

Lemma 9 Let $G$ be an imprimitive group of degree $p^{2}$ with Sylow p-subgroup isomorphic to $P_{p-1}^{\prime}$. Let $\mathcal{B}$ be the unique complete block system of $P_{p-1}^{\prime}$ of $p$ blocks of cardinality $p$. Then there exists a subgroup $H \leq S_{p} \times \operatorname{AGL}(1, p)$ such that $G$ is equivalent to $H \cdot \operatorname{fix}_{P_{p-1}^{\prime}}(\mathcal{B})$.

Proof: We assume without loss of generality that a Sylow $p$-subgroup of $G$ is $P_{p-1}^{\prime}$ so that $G$ admits $\mathcal{B}$ as a complete block system. As $P_{p-1}^{\prime}$ is a Sylow $p$-subgroup of $G, C_{\mathcal{B}}$ is nondegenerate. It then follows by Lemma 7 that $\operatorname{fix}_{G}(\mathcal{B})$ is solvable and hence $\left.\operatorname{fix}_{G}(\mathcal{B})\right|_{B} \leq$ $\operatorname{AGL}(1, p)$ for every $B \in \mathcal{B}$. We now show that if $g \in G$, then $g \in N_{S_{p^{2}}}\left(\left\langle\tau_{2}\right\rangle\right)$.

As $\left.\operatorname{fix}_{G}(\mathcal{B})\right|_{B} \leq \operatorname{AGL}(1, p)$ for every $B \in \mathcal{B}$ if $g \in G$, then $g(i, j)=\left(\sigma(i), \alpha_{i} j+b_{i}\right)$, where $\sigma \in S_{p}, \alpha_{i} \in \mathbb{F}_{p}^{*}$ and $b_{i} \in \mathbb{Z}_{p}$. To show that $g \in N_{S_{p^{2}}}\left(\left\langle\tau_{2}\right\rangle\right)$ is suffices to show that $\alpha_{i}=\alpha_{j}$ for every $i, j \in \mathbb{Z}_{p}$. As a Sylow $p$-subgroup of $G$ is $P_{p-1}^{\prime}$, by Lemma $4 C_{\mathcal{B}}$ consists of all vectors in $\mathbb{F}_{p}^{p}$ such that the sum of the coordinates is congruent to 0 modulo $p$. We conclude that $z_{i} z_{j}^{p-1} \in \operatorname{fix}_{G}(\mathcal{B})$ for every $i \neq j \in \mathbb{Z}_{p}$. Then $g z_{i} z_{j}^{p-1} g^{-1}=z_{\sigma(i)}^{\alpha_{i}} z_{\sigma(j)}^{\alpha_{j}(p-1)}$. As $g z_{i} z_{j}^{p-1} g^{-1} \in \operatorname{fix}_{G}(\mathcal{B})$, we must have that $\alpha_{i}+\alpha_{j}(p-1) \equiv 0(\bmod p)$, so that $\alpha_{i}=\alpha_{j}$. Thus if $g \in G$, then $g \in N_{S_{p^{2}}}\left(\left\langle\tau_{2}\right\rangle\right)$. 
Let $B \in \mathcal{B}$ and $L=\{g \in G: g(B)=B\}$. Then $L$ has a unique Sylow $p$-subgroup $P$, namely the Sylow $p$-subgroup of $\operatorname{fix}_{G}(\mathcal{B})$, so that $P \triangleleft L$. By the Schur-Zassenhaus Theorem [14, Theorem 2.1, p. 221] $L$ contains a $p^{\prime}$-subgroup $M$ which is a complement to $P$ in $L$. Let $P^{\prime}=\left\langle z_{i}: i \in \mathbb{Z}_{p}\right\rangle$ and $W=G \cdot P^{\prime}$. Note that $|W|=p \cdot|G|$, and let $K=\{w \in W: w(B)=B\}$. Then $P^{\prime} \triangleleft K$ and is the Sylow $p$-subgroup of $K$. Again by the Schur-Zassenhaus Theorem, as $P^{\prime}$ is solvable, any two $p^{\prime}$-subgroups of $K$ are conjugate in $W$. As $M$ is a $p^{\prime}$-subgroup of $L, M$ is a $p^{\prime}$-subgroup of $K$. Recall that if $g \in G$, then $g(i, j)=\left(\sigma(i), \alpha j+b_{i}\right), \sigma \in S_{p}, \alpha \in \mathbb{F}_{p}^{*}$, and $b_{i} \in \mathbb{Z}_{p}$. For $g \in G$, define $\hat{g}: S_{\mathbb{Z}_{p}^{2}} \rightarrow S_{\mathbb{Z}_{p}^{2}}$ by $\hat{g}(i, j)=(\sigma(i), \alpha j)$, and let $\hat{M}=\{\hat{g}: g \in M\}$. Clearly $\hat{M}$ is a subgroup and $|\hat{M}|=|M|$. Furthermore, as $P^{\prime}=\left\langle z_{i}: i \in \mathbb{Z}_{p}\right\rangle, \hat{M} \leq K$. Thus $\hat{M}$ is also a $p^{\prime}$ subgroup of $K$ so that there exists $\delta \in P^{\prime}$ such that $\delta^{-1} M \delta=\hat{M}$. Let $G^{\prime}=\delta^{-1} G \delta$. Clearly $\hat{M} \leq G^{\prime}$ and as $\delta \in P^{\prime} \leq N_{S_{p^{2}}}\left(P_{p-1}^{\prime}\right)$ we have that $P_{p-1}^{\prime} \leq G^{\prime}$. Let $H=\left\langle\tau_{1}, \hat{M}\right\rangle$. Then $H \leq G^{\prime}$ and for every $h \in H, h(i, j)=(\sigma(i), \alpha j)$ so that $H \leq S_{p} \times \operatorname{AGL}(1, p)$. As $|M|=|G| / p^{p}$ we have that $|H|=|G| / p^{p-1}$ so that $\left|H \cdot \operatorname{fix}_{P_{p-1}^{\prime}}(\mathcal{B})\right|=|G|=\left|G^{\prime}\right|$. As $H \cdot \operatorname{fix}_{P_{p-1}^{\prime}}(\mathcal{B}) \leq G^{\prime}$, we conclude that $G^{\prime}=H \cdot \operatorname{fix}_{P_{p-1}^{\prime}}(\mathcal{B})$ and the result follows.

Proof of Theorem 4: (1) Follows from Lemma 6, and (2) follows from Theorem 8.

Thus, we assume, henceforth, that $G$ is imprimitive. By Theorem 9 the Sylow $p$-subgroups of $G$ are isomorphic to $P_{i}$ or $P_{i}^{\prime}, 1 \leq i \leq p$. If no Sylow $p$-subgroup of $G$ is isomorphic to $P_{1}^{\prime}$ or $P_{p-1}^{\prime}$, then (3) follows from Theorem 3. If a Sylow $p$-subgroup of $G$ is isomorphic to $P_{1}^{\prime}$, then (4) follows from Theorem 7 and Lemma 1. Finally, if a Sylow $p$-subgroup of $G$ is isomorphic to $P_{p-1}^{\prime}$, then (5) follows from Lemma 9.

\section{Imprimitive subgroups that contain a Sylow $p$-subgroup of $S_{p^{2}}$}

Note that $\mathbb{Z}_{p} 2 \mathbb{Z}_{p}$ is a Sylow $p$-subgroup of $S_{p^{2}}$.

Proof of Proposition 1: $\quad(\Rightarrow)$ Because $N_{S_{p}}(L) / L$ is cyclic (see Theorem 2), we know that $\left(N_{S_{p}}(L) / L\right)^{p}$ is abelian, so it is obvious that $K / L^{p}$ is a normal subgroup of $\left(N_{S_{p}}(L) / L\right)^{p}$; hence $K$ is a normal subgroup of $N_{S_{p}}(L)^{p}$. Then, because $\phi$ is a crossed homomorphism and $K$ is $H$-invariant, it is easy to verify that $G_{H, L, K, \phi}$ is closed under multiplication. Therefore, it is a subgroup of $S_{p} 2 S_{p}$.

It is straightforward to verify that $K$ is a normal subgroup of $G_{H, L, K, \phi}$, and we have $G_{H, L, K, \phi} / K \cong H$, so $\left|G_{H, L, K, \phi}\right|$ is divisible by $|K||H|$. Because $K \supset L^{p}$, this implies that $\left|G_{H, L, K, \phi}\right|$ is divisible by $p^{p+1}$. Therefore, $G_{H, L, K, \phi}$ contains a Sylow $p$-subgroup of $S_{p} 2 S_{p}$, so $G_{H, L, K, \phi}$ is transitive. Because $G_{H, L, K, \phi} \leq S_{p} 2 S_{p}$, we know that $G_{H, L, K, \phi}$ is imprimitive.

$(\Leftarrow)$ Because $G$ is imprimitive, we may assume that $G \leq S_{p} 2 S_{p}$. Then, because $p^{p+1}$ | $|G|$, we know that $G$ contains a Sylow $p$-subgroup of $S_{p} 2 S_{p}$; assume, without loss of generality, that $G$ contains $\mathbb{Z}_{p} 2 \mathbb{Z}_{p}$. In particular, $G$ admits a unique block system $\mathcal{B}$, consisting of $p$ blocks of cardinality $p$.

Let $H=G / \mathcal{B} \leq S_{p}$, let $K=\mathrm{fix}_{G}(\mathcal{B})$, and let $\hat{L}$ be the smallest normal subgroup of $G$ that contains $1 \gtrless \mathbb{Z}_{p}$. It is easy to see that $\hat{L}=1 \imath L \cong L^{p}$, for some transitive, simple subgroup $L$ of $S_{p}$. 
The map $g \mapsto g / \mathcal{B}$ is a homomorphism from $G$ onto $H$, with kernel $K$. Thus, there is an isomorphism $\hat{\phi}: H \rightarrow G / K$, given by $h=\hat{\phi}(h) / \mathcal{B}$. Because 1 乙 $L$ is normal in $G$, we know that $G \leq H \geq N_{S_{p}}(L)$, so we may write $\hat{\phi}(h)=h \phi(h)$, with $\phi(h) \in\left(12 N_{S_{p}}(L)^{p}\right) / K$. Because $\hat{\phi}$ is a homomorphism, it is straightforward to verify that $\phi$ is a crossed homomorphism.

The assumption that $L$ is simple is not necessary in the definition of $G_{H, L, K, \phi}$, but this restriction makes $L$ unique (up to conjugacy). For a given group $G$, the corresponding $H, L, K, \phi$ are not uniquely determined, but the following simple lemma describes how to tell whether $G_{H_{1}, L_{1}, K_{1}, \phi_{1}}$ is equivalent to $G_{H_{2}, L_{2}, K_{2}, \phi_{2}}$.

Definition 10 (cf. [9, Proposition 4.1]) Let $H$ be a group, let $A$ be an $H$-module, and let $\phi_{1}, \phi_{2}: H \rightarrow A$ be crossed homomorphisms. We say that $\phi_{1}$ is cohomologous to $\phi_{2}$ if there is an element $a$ of $A$, such that, for every $h \in H$, we have

$$
\phi_{1}(h)-\phi_{2}(h)=h^{-1} a-a .
$$

(This is equivalent to the assertion that the homomorphisms $h \mapsto\left(h, \phi_{1}(h)\right)$ and $h \mapsto$ $\left(h, \phi_{1}(h)\right)$ are conjugate via an element of $A$.)

We remark that the equivalence classes of this equivalence relation are, by definition, the elements of the cohomology group $H^{1}(H, A)$.

Lemma 10 Let $H_{i}, L_{i}, K_{i}, \phi_{i}$ be as in Proposition 1 , for $i=1,2$.

1. If $G_{H_{1}, L_{1}, K_{1}, \phi_{1}}$ is equivalent to $G_{H_{2}, L_{2}, K_{2}, \phi_{2}}$, then $L_{1}$ is conjugate to $L_{2}$ (in $S_{p}$ ).

2. If $G_{H_{1}, L_{1}, K_{1}, \phi_{1}}$ is equivalent to $G_{H_{2}, L_{2}, K_{2}, \phi_{2}}$, and $L_{1}=L_{2}$, then there exists $g \in S_{p}$, such that, letting $\hat{g}=(g, 1) \in S_{p} 2 S_{p}$, we have

(A) $\mathrm{gH}_{1} \mathrm{~g}^{-1}=\mathrm{H}_{2}$;

(B) $\hat{g} K_{1} \hat{g}^{-1}=K_{2}$; and

(C) $\phi_{1}^{g}$ is cohomologous to $\phi_{2}$, where $\phi_{1}^{g}: H_{2} \rightarrow\left(N_{S_{p}}\left(L_{2}\right) / L_{2}\right)^{p}$ is defined by $\phi_{1}^{g}(h)=$ $\hat{g} \phi_{1}\left(g^{-1} h g\right) \hat{g}^{-1}$.

Proof: Let $h \in S_{p^{2}}$, with $h G_{H_{1}, L_{1}, K_{1}, \phi_{1}} h^{-1}=G_{H_{2}, L_{2}, K_{2}, \phi_{2}}$, and let $\mathcal{B}$ be the unique complete block system for $\mathbb{Z}_{p} 2 \mathbb{Z}_{p}$. Because $\mathbb{Z}_{p} 2 \mathbb{Z}_{p}$ is contained in both $G_{H_{1}, L_{1}, K_{1}, \phi_{1}}$ and $G_{H_{2}, L_{2}, K_{2}, \phi_{2}}$, the uniqueness of $\mathcal{B}$ implies that $h \mathcal{B}=\mathcal{B}$; thus, $h \in S_{p} 2 S_{p}$, so we may write $h=(g, x)$, with $g \in S_{p}$ and $x \in\left(S_{p}\right)^{p}$. Because $L_{1}=L_{2}$, we must have $x \in N_{S_{p}}\left(L_{2}\right)^{p}$.

Because $N_{S_{p}}\left(L_{2}\right) / L_{2}$ is abelian, this implies that $x$ normalizes $K_{2}$, so we must have $g K_{1} g^{-1}=K_{2}$.

Because $H_{i}=G_{H_{i}, L_{i}, K_{i}, \phi_{i}} / \mathcal{B}$, we must have $g H_{1} g^{-1}=H_{2}$.

Replacing $G_{H_{1}, L_{1}, K_{1}, \phi_{1}}$ by its conjugate under $\hat{g}$, we may assume that $g=1$, so $H_{1}=H_{2}$, $K_{1}=K_{2}$, and $\phi_{1}^{g}=\phi_{1}$. Because

$$
\begin{aligned}
x\left\{\left(h, \phi_{1}(h)\right): h \in H_{1}\right\} x^{-1} & =\frac{x G_{H_{1}, L_{1}, K_{1}, \phi_{1}} x^{-1}}{L_{1}}=\frac{G_{H_{2}, L_{2}, K_{2}, \phi_{2}}}{L_{2}} \\
& =\left\{\left(h, \phi_{2}(h)\right): h \in H_{2}\right\}
\end{aligned}
$$

we see that $\phi_{1}$ is cohomologous to $\phi_{2}$. 


\subsection{Cyclic codes modulo $n$}

The Chinese Remainder Theorem (Lemma 11) reduces the study of codes modulo $n$ to the case where $n$ is a prime power. Assuming that $p \nmid n$, the problem can often be further reduced to the case where $n$ is prime (see Lemma 12 and Remark 11). This reduced case is considered in Lemma 13.

Lemma 11 (cf. [14, Theorem 1.2.13, p. 8]) Let $n=n_{1} n_{2} \ldots n_{r}$, where each $n_{i}$ is a prime power, and $n_{1}, n_{2}, \ldots, n_{r}$ are pairwise relatively prime.

1. We have $\left(\mathbb{Z}_{n}\right)^{p} \cong\left(\mathbb{Z}_{n_{1}}\right)^{p} \oplus\left(\mathbb{Z}_{n_{2}}\right)^{p} \oplus \cdots \oplus\left(\mathbb{Z}_{n_{r}}\right)^{p}$.

2. For any subgroup $C$ of $\left(\mathbb{Z}_{n_{1}}\right)^{p} \oplus\left(\mathbb{Z}_{n_{2}}\right)^{p} \oplus \cdots \oplus\left(\mathbb{Z}_{n_{r}}\right)^{p}$, we have

$$
C=\left(C \cap\left(\mathbb{Z}_{n_{1}}\right)^{p}\right) \oplus\left(C \cap\left(\mathbb{Z}_{n_{2}}\right)^{p}\right) \oplus \cdots \oplus\left(C \cap\left(\mathbb{Z}_{n_{r}}\right)^{p}\right) .
$$

Definition 11 If $n=q^{t}$, where $q$ is prime, and $0 \leq i<t$, we let $\phi_{i}: q^{i}\left(\mathbb{Z}_{n}\right)^{p} \rightarrow\left(\mathbb{Z}_{q}\right)^{p}$ be the natural homomorphism with kernel $q^{i+1}\left(\mathbb{Z}_{n}\right)^{p}$.

Lemma 12 Let $n=q^{t}$ where $q$ is prime, and $p \neq q$, and let $G$ be any transitive group of degree $p$ that contains $\mathbb{Z}_{p}$.

1. If $C$ is any $G$-invariant subgroup of $\left(\mathbb{Z}_{n}\right)^{p}$, define $C_{i}=\phi_{i}\left(C \cap q^{i}\left(\mathbb{Z}_{n}\right)^{p}\right)$ for $0 \leq i<t$. Then $C_{0} \subset C_{1} \subset \cdots \subset C_{t-1}$ is an increasing chain of $G$-invariant subgroups of $\left(\mathbb{Z}_{q}\right)^{p}$.

2. If $G \leq \operatorname{AGL}(1, p)$, or $G=A_{n}$, or $G=S_{n}$, then the converse holds: For any increasing chain $C_{0} \subset C_{1} \subset \cdots \subset C_{t-1} \subset\left(\mathbb{Z}_{q}\right)^{p}$ of $G$-invariant subgroups of $\left(\mathbb{Z}_{q}\right)^{p}$, there is a subgroup of $\left(\mathbb{Z}_{n}\right)^{p}$, such that $\phi_{i}\left(C \cap q^{i}\left(\mathbb{Z}_{n}\right)^{p}\right)=C_{i}$, for $0 \leq i<t$.

3. Each $G$-invariant subgroup of $\left(\mathbb{Z}_{n}\right)^{p}$ is uniquely determined by the corresponding chain $C_{0} \subset C_{1} \subset \cdots \subset C_{t-1}$ of $G$-invariant subgroups of $\left(\mathbb{Z}_{q}\right)^{p}$.

Proof: (1) This follows from the observation that, for any $c \in C \cap q^{i}\left(\mathbb{Z}_{n}\right)^{p}$, we have $q c \in q^{i+1}\left(\mathbb{Z}_{n}\right)^{p}$ and $\phi_{i}(c)=\phi_{i+1}(q c)$.

(3) Suppose there is a code $C^{\prime}$, such that $C_{i}^{\prime}=C_{i}$ for each $i$. Let $M=C \cap q\left(\mathbb{Z}_{n}\right)^{p}$. By induction on $t$, we may assume that $C^{\prime} \cap q\left(\mathbb{Z}_{n}\right)^{p}=M$. Consider the composite homomorphism:

$$
C_{0} \cong \frac{C}{M} \hookrightarrow \frac{C^{\prime}+q\left(\mathbb{Z}_{n}\right)^{p}}{M} \rightarrow \frac{C^{\prime}+q\left(\mathbb{Z}_{n}\right)^{p}}{C^{\prime}} \cong \frac{q\left(\mathbb{Z}_{n}\right)^{p}}{M}
$$

If $C \neq C^{\prime}$, then this homomorphism is nontrivial, so $C_{0}$ and $q\left(\mathbb{Z}_{n}\right)^{p} / M$ have a composition factor in common. Because

$$
M \subset M+q^{t-1}\left(\mathbb{Z}_{n}\right)^{p} \subset M+q^{t-2}\left(\mathbb{Z}_{n}\right)^{p} \cdots \subset M+q\left(\mathbb{Z}_{n}\right)^{p}
$$

is an increasing chain of $G$-submodules with quotients

$$
\left(\mathbb{Z}_{q}\right)^{p} / C_{t-1},\left(\mathbb{Z}_{q}\right)^{p} / C_{t-2}, \ldots,\left(\mathbb{Z}_{q}\right)^{p} / C_{1},
$$


we conclude that $C_{0}$ has a composition factor in common with $\left(\mathbb{Z}_{q}\right)^{p} / C_{i}$, for some $i \geq 1$. This is impossible, because $C_{0} \subset C_{i}$, and the representation of $G$ on $\left(\mathbb{Z}_{q}\right)^{p}$ is multiplicity free. (In fact, the restriction to the subgroup $\mathbb{Z}_{p}$ is multiplicity free, because there are $p$ distinct $p$ th roots of unity in an appropriate extension of $\mathbb{F}_{q}$.)

(2) It suffices to show, for each $i$, that there is a $G$-invariant subgroup $\hat{C}_{i}$ of $\left(\mathbb{Z}_{n}\right)^{p}$, such that $\hat{C}_{j}=C_{i}$ for $j \leq i$ and $\hat{C}_{j}=0$ for $j>i$. (For then we simply let $C=\left\langle\hat{C}_{0}, \ldots, \hat{C}_{t-1}\right\rangle$.) Thus, we may assume that, for some $i$, we have $C_{0}=C_{1}=\cdots=C_{i}$ and $C_{i+1}=C_{i+2}=$ $\cdots=C_{t-1}=0$. Furthermore, may assume that $i=t-1$ (because $C^{\prime}=q^{k} C$ satisfies $C_{j}^{\prime}=0$ for $j \geq t-k$ ).

If $C_{i}$ is the repetition code, let $C$ be the repetition code in $\left(\mathbb{Z}_{n}\right)^{p}$. If $C_{i}$ is the dual of the repetition code, then let $C$ be the dual of the repetition code in $\left(\mathbb{Z}_{n}\right)^{p}$; that is,

$$
C=\left\{\left(z_{1}, \ldots, z_{p}\right) \in\left(\mathbb{Z}_{n}\right)^{p}: \sum_{i=1}^{p} z_{i} \equiv 0 \quad(\bmod n)\right\}
$$

Thus, we may now assume that $C_{i}$ is neither the repetition code nor its dual. Then, from Lemma 13 and the assumption on $G$, we see that $G \leq \operatorname{AGL}(1, p)$. Therefore $\mathbb{Z}_{p} \triangleleft G$, so, from uniqueness (3), we see that every $\mathbb{Z}_{p}$-invariant subgroup of $\left(\mathbb{Z}_{n}\right)^{p}$ is $G$-invariant. Thus, we may assume that $G=\mathbb{Z}_{p}$.

In this case, the desired conclusion is a special case of [7, Theorem 37.4, p. 156], but we give an explicit construction. Let $f(x) \in \mathbb{F}_{q}[x]$ be the monic generating polynomial for $C_{i}$. Because $f(x)$ is a divisor of $x^{p}-1$, and $x^{p}-1$ has no repeated roots, we know, from Hensel's Lemma $\left[7,36.5\right.$, p. 145], that there is a monic polynomial $g(x) \in \mathbb{Z}_{n}[x]$, such that $g(x) \equiv f(x)(\bmod q), \operatorname{deg}(g)=\operatorname{deg}(f)$, and $g$ is a divisor of $x^{p}-1$ in $\mathbb{Z}_{n}[x]$. Now let $C$ be the ideal of $\mathbb{Z}_{n}[x] /\left(x^{p}-1\right)$ generated by $g(x)$.

Remark In applying Lemma 12 to the study of subgroups of $S_{p^{2}}$, one is interested only in the case where $n$ is not prime and there is a subgroup $L$ of $S_{p}$, such that $n$ is a divisor of $\left|N_{S_{p}}(L) / L\right|$. Note that $3^{2} \nmid 11-1,2^{2} \nmid 23-1$, and neither 11 nor 23 can be written in the form $\left(q^{d}-1\right) /(q-1)$ for a prime-power $q$. Therefore, we see from Lemma 13 that if $G=\operatorname{PSL}(2,11), M_{11}$, or $M_{23}$, then, in the cases of interest, $C_{i}$ must be either the repetition code or its dual. Thus, the proof of Lemma 12 is valid in these cases. It is only when $\operatorname{PSL}(d, q) \leq G \leq \operatorname{P\Gamma L}(d, q)$ that the possible choices of $K$ in Proposition 1(3) have not yet been completely classified.

Lemma $13([3,23,29]) \quad$ Let

- $p$ and $r$ be prime;

- $G$ be a transitive subgroup of $S_{p}$ that contains $\mathbb{Z}_{p}$, and

- $C$ be a nontrivial cyclic code over $\mathbb{Z}_{r}$ that admits $G$ as a group of permutation automorphisms.

If $C$ is neither the repetition code nor its dual, then either

1. $\mathbb{Z}_{p} \leq G \leq \mathrm{AGL}(1, p)$, and $C$ is described in Lemma 14 below; or

2. $G=\operatorname{PSL}(2,11), p=11, r=3$, and $C$ is either the $(11,6)$ ternary Golay code or its dual; or 
3. $G=M_{23}, p=23, r=2$, and $C$ is either the $(23,12)$ binary Golay code or its dual; or

4. $\operatorname{PSL}(d, q) \leq G \leq \operatorname{P\Gamma L}(d, q), p=\left(q^{d}-1\right) /(q-1), q$ is a power of $r$, and $C$ is described in Theorem 12 below.

Proof: From Theorem 2, we know that there are only a few possibilities for $G$. In each case, the desired conclusion is a known result.

- If $\mathbb{Z}_{p} \leq G \leq \operatorname{AGL}(1, p)$, see Lemma 14 .

- If $G=A_{p}\left(\right.$ or $\left.S_{p}\right)$, see [23, Beispiele 9(a)].

- If $G=\operatorname{PSL}(2,11)$ and $p=11$, see [29, (J)].

- If $G=M_{11}$ or $M_{23}$ (and $p=11$ or 23, respectively), see [23, Beispiele 9(bc)].

- Suppose $\operatorname{PSL}(d, q) \leq G \leq \operatorname{P\Gamma L}(d, q)$ and $p=\left(q^{d}-1\right) /(q-1)$. If $r \nmid q$, see [29, Section 3(C)]; if $r \mid q$, see Theorem 12.

5.1.1. Cyclic codes invariant under a given subgroup of AGL $(1, p)$. Lemma 14 characterizes the cyclic codes of prime length $p$ that admit a given subgroup of AGL $(1, p)$ as permutation automorphisms. This result must be well known, but the authors have been unable to locate it in the literature.

Lemma 14 Let $f(x) \in \mathbb{F}_{q}[x]$ be the generating polynomial of a cyclic code $C$ of prime length $p$ over $\mathbb{F}_{q}$, and let $A$ be a subgroup of $\mathbb{Z}_{p}^{*}$.

1. If $p \nmid q$, then $C$ is $A$-invariant if and only if $f(x)$ is a factor of $f\left(x^{a}\right)$, for every $a \in A$.

2. If $p \mid q$, then $C$ is $A$-invariant.

Remark Suppose $p \nmid q$. For a given subgroup $A$ of $\mathbb{Z}_{p}^{*}$, one can construct all of the $A$-invariant cyclic codes of length $p$ by the following method.

Let $\mathcal{P} \subset \mathbb{F}_{q}[x]$ be the set of all monic factors of the polynomial $x^{p}-1$, and let $\mathcal{P}_{\text {irr }}$ be the subset consisting of those polynomials that are irreducible over $\mathbb{F}_{q}$. Then $A$ acts on both $\mathcal{P}$ and $\mathcal{P}$ irr by

$$
f^{a}(x)=\operatorname{gcd}\left(f\left(x^{a}\right), x^{p}-1\right) .
$$

From the lemma, we see that $f(x)$ is the generating polynomial of an $A$-invariant code if and only if $f^{a}=f$, for every $a \in A$.

If $F$ is any $A$-invariant subset $F$ of $\mathcal{P}_{\text {irr }}$ (that is, if $F$ is any union of orbits of $A$ ), then $\prod_{f \in F} f(x)$ is the generating polynomial of an $A$-invariant code, and conversely, every $A$-invariant generating polynomial can be constructed in this way.

In particular, the number of $A$-invariant cyclic codes is $2^{d}$, where $d$ is the number of $A$-orbits on $\mathcal{P}_{\text {irr }}$. However, it is probably easier to calculate $d$ by using the formula $d=$ $1+\left|\mathbb{Z}_{p}^{*}:\langle A, q\rangle\right|$.

5.1.2. Codes that admit PSL $(\boldsymbol{d}, \boldsymbol{q})$. Bardoe and Sin [3, Theorem A] recently gave an explicit description of the codes that admit $\operatorname{PGL}(d, q)$ as a group of permutation automorphisms. (They [3, Theorem C] also considered monomial automorphisms, but we do not need the more general result.) For the case of interest to us, where $\left(q^{d}-1\right) /(q-1)$ is prime, 
we know that $\operatorname{gcd}(q-1, d)=1$, so the natural embedding of $\operatorname{PSL}(d, q)$ into $\operatorname{PGL}(d, q)$ is an isomorphism. Therefore, the codes described in [3] are precisely the codes that admit $\operatorname{PSL}(d, q)$ as a group of permutations.

Furthermore, the results of Bardoe and Sin yield an explicit description of the image of each code under the Frobenius automorphism (cf. [3, Theorem A(b)]), so the results generalize easily to any subgroup $G$ of $\operatorname{P\Gamma L}(d, q)$ that contains $\operatorname{PSL}(d, q)$. After some necessary definitions, we state this slightly more general version of [3, Theorem A].

Definition 12 Suppose $r$ is a prime number, $q=r^{t}$, and $p=\left(q^{d}-1\right) /(q-1)$ is prime. Let $c$ be a divisor of $t$.

Let $\mathcal{H}^{(c)}$ denote the set of $t$-tuples $\left(s_{0}, s_{1}, \ldots, s_{t-1}\right)$ of integers satisfying (for $j=$ $0,1, \ldots, t-1$, and with subscripts read modulo $t)$ :

1. $1 \leq s_{j} \leq d-1$;

2. $0 \leq r s_{j+1}-s_{j} \leq(r-1) d$; and

3. $s_{j+c}=s_{j}$.

Let $\mathcal{H}^{(c)}$ be partially ordered in the natural way: $\left(s_{0}^{\prime}, \ldots, s_{t-1}^{\prime}\right) \leq\left(s_{0}, \ldots, s_{t-1}\right)$ if and only if $s_{j}^{\prime} \leq s_{j}$ for all $j$.

Let $\mathcal{H}_{0}^{(c)}=\mathcal{H}^{(c)} \cup\{(0,0, \ldots, 0)\}$, and extend the partial order on $\mathcal{H}^{(c)}$ to $\mathcal{H}_{0}^{(c)}$, by making $(0,0, \ldots, 0)$ incomparable to all other elements.

Definition 13 A monomial $X=\prod_{i=1}^{d} X_{i}^{b_{i}} \in \mathbb{Z}_{r}\left[X_{1}, X_{2}, \ldots, X_{d}\right]$ is a basis monomial if

- $0 \leq b_{i}<q$, for $i=1, \ldots, d$;

- $\operatorname{deg}(X)=\sum_{i=1}^{d} b_{i}$ is divisible by $q-1$; and

- $X \neq X_{1}^{q-1} X_{2}^{q-1} \ldots X_{d}^{q-1}$.

Definition 14 ([3, Section 3.2]) Let $X=\prod_{i=1}^{d} X_{i}^{b_{i}}$ be a basis monomial. For each $e \in\{0,1, \ldots, t-1\}$, let

$$
\operatorname{deg}^{e}(X)=\sum_{i=1}^{d} \phi^{e}\left(b_{i}\right),
$$

where $\phi$ is the permutation on $\{0,1, \ldots, q-1\}$ defined by $\phi(k)=r k+(1-q)\lfloor r k / q\rfloor$. (In other words, if we write $k=\sum_{j=0}^{t-1} a_{j} r^{j}$ as a $t$-digit number in base $r$, then $\phi(k)=$ $a_{t-1}+\sum_{j=1}^{t-1} a_{j-1} r^{j}$ is the $t$-digit number obtained by rotating the $t$ digits of $k$, including the leading 0's.)

Define

$$
s(X)=\frac{1}{q-1}\left(\operatorname{deg}^{0}(X), \operatorname{deg}^{1}(X), \ldots, \operatorname{deg}^{t-1}(X)\right) .
$$

Then $s(X) \in \mathcal{H}_{0}^{(t)}$. 
Definition 15 Any basis monomial $X$ defines an $\mathbb{F}_{q}$-valued function $f_{X}$ on the vector space $\mathbb{F}_{q}^{d}$. Because $\operatorname{deg}(X)$ is divisible by $q-1$, we have $f_{X}(v)=f_{x}(\lambda v)$, for every $\lambda \in \mathbb{F}_{q}^{*}$ and $v \in \mathbb{F}_{q}^{d}$, so $f_{X}$ factors through to a well-defined function $\bar{f}_{X}$ on the projective space $\mathbb{P}^{d-1}\left(\mathbb{F}_{q}\right)$.

Theorem 12 (Bardoe-Sin [3]) Suppose $r$ is a prime number, $q=r^{t}, p=\left(q^{d}-1\right) /(q-1)$ is prime, and $\operatorname{PSL}(d, q) \leq G \leq \operatorname{P\Gamma L}(d, q)$. Let $c=|\operatorname{P\Gamma L}(d, q): G|$.

For any ideal $\mathcal{I}$ of the partially ordered set $\mathcal{H}_{0}^{(c)}$, let $M_{\mathcal{I}} \subset \mathbb{Z}_{r}\left[\mathbb{P}^{d-1}\left(\mathbb{F}_{q}\right)\right]$ be the span over $\mathbb{Z}_{r}$ of the functions $\bar{f}_{X}$, for all basis monomials $X$, such that $s(X) \in \mathcal{I}$. Then $M_{\mathcal{I}}$ is $G$-invariant.

Conversely, for each $G$-invariant subspace $M$, there is a unique ideal $\mathcal{I}$ of $\mathcal{H}_{0}^{(c)}$, such that $M=M_{\mathcal{I}}$.

\subsection{Crossed homomorphisms}

Theorem 13 Let

- $p$ be a prime;

- $H$ be either $A_{p}, S_{p}$, or subgroup of $\operatorname{AGL}(1, p)$ that contains $\mathbb{Z}_{p}$;

- $n$ be a natural number, such that either $n=2$ or $n \mid p-1$ or $n \mid m$, where $m$ satisfies $p=\left(r^{d^{m}}-1\right) /\left(r^{d}-1\right)$ for some prime $r$ and natural number $d$

- $K$ be an $H$-invariant subgroup of $\left(\mathbb{Z}_{n}\right)^{p}$; and

- $\phi: H \rightarrow\left(\mathbb{Z}_{n}\right)^{p} / K$ be a crossed homomorphism.

Then $\phi$ is cohomologous to a homomorphism from $H$ to $C_{0} /\left(K \cap C_{0}\right)$, where $C_{0}$ is the repetition code in $\left(\mathbb{Z}_{n}\right)^{p}$.

Remark The conclusion of the theorem can be stated more concretely: If $\phi$ is not cohomologous to 0 , then either

1. $H \leq \operatorname{AGL}(1, p)$, and there is some $c \in \mathbb{Z}_{n}$, and some generator $h$ of $H / \mathbb{Z}_{p}$, such that $|h|(c, c, \ldots, c) \in K$ and, after replacing $\phi$ by a cohomologous cocycle, we have $\phi\left(h^{a}, z\right)=a(c, c, \ldots, c)$, for $a \in \mathbb{Z}$ and $z \in \mathbb{Z}_{p} ;$ or

2. $H=S_{p}, n$ is even, and there is some $c \in \mathbb{Z}_{n}$, such that $(2 c, 2 c, \ldots, 2 c) \in K$ and, after replacing $\phi$ by a cohomologous cocycle, we have

$$
\phi(h)= \begin{cases}0+K & \text { if } g \in A_{p} \\ (c, c, \ldots, c)+K & \text { if } g \notin A_{p}\end{cases}
$$

Proof: Let $V=\left(\mathbb{Z}_{n}\right)^{p} / K$, let, and let $C_{0}^{\perp}$ be its dual.

Because $\operatorname{gcd}(p, n)=1$, we know that every element of $C_{V}\left(\mathbb{Z}_{p}\right)$ has a representative in $C_{\left(\mathbb{Z}_{n}\right)^{p}}\left(\mathbb{Z}_{p}\right)$ (cf. [14, Theorem 5.2.3, p. 177]). Therefore

$$
C_{V}(H) \subset C_{V}\left(\mathbb{Z}_{p}\right)=\frac{C_{\left(\mathbb{Z}_{n}\right)^{p}}\left(\mathbb{Z}_{p}\right)+K}{K}=\frac{C_{0}+K}{K} \cong \frac{C_{0}}{K \cap C_{0}} .
$$


Thus, it suffices to show that, after replacing $\phi$ by a cohomologous crossed homomorphism, we have $\phi(H) \subset C_{V}\left(\mathbb{Z}_{p}\right)$.

Case 1 Assume $H \leq \operatorname{AGL}(1, p)$. Because $\operatorname{gcd}(p, n)=1$, we know that $H^{1}\left(\mathbb{Z}_{p}, V\right)=0$ [9, Corollary 12.2 .7$, p. 237]. Therefore, replacing $\phi$ by a cohomologous cocycle, we may assume that $\phi\left(\mathbb{Z}_{p}\right)=0$. Because $\phi$ is a crossed homomorphism, this implies that $\phi(H) \subset$ $C_{V}\left(\mathbb{Z}_{p}\right)$.

Case 2 Assume $H=A_{p}$. Assume that $n$ is prime. From Lemma 13, we know that $\left(\mathbb{Z}_{n}\right)^{p} / K$ is either $C_{0}$ or $C_{0}^{\perp}$. Because $A_{p}$ is perfect (or $p=3$, in which case $A_{p}=\mathbb{Z}_{p}$ ), we know that $H^{1}\left(A_{p}, C_{0}\right)=0$. From [24, Lemma 1], we know that $H^{1}\left(A_{p}, C_{0}^{\perp}\right)=0$.

Let $m$ be a divisor of $n$, such that $n / m$ is prime. By induction on $n$, we may assume that $\phi$ is cohomologous to a crossed homomorphism into $m V$. Then the preceding paragraph implies that $\phi$ is cohomologous to 0 .

Case 3 Assume $H=S_{p}$. From Case 2, we may assume, after replacing $\phi$ by a cohomologous crossed homomorphism, that $\phi\left(A_{p}\right)=0$. Therefore, $\phi\left(S_{p}\right) \subset C_{V}\left(A_{p}\right)=C_{V}\left(\mathbb{Z}_{p}\right)$.

Remark To complete the classification of transitive subgroups of $S_{p^{2}}$, the following problems remain:

- For PSL $(d, q) \leq G \leq \operatorname{PLL}(d, q)$, extend the Bardoe-Sin Theorem 12 from a classification of subgroups modulo a prime to a classification modulo a prime-power.

- Calculate $H^{1}(H, V)$ for $H=\operatorname{PSL}(2,11)$ (with $\left.p=11\right), M_{11}, M_{23}$ and $\operatorname{PSL}(d, q)$.

- For each nontrivial cohomology class, find an explicit crossed homomorphism to represent it.

\section{Applications}

\subsection{The Cayley isomorphism problem}

Let $H$ be a set, and $E \subseteq 2^{H} \cup 2^{2^{H}} \cup \ldots$. We say that the ordered pair $X=(H, E)$ is a combinatorial object. We call $H$ the vertex set and $E$ the edge set. If $E \subseteq 2^{H}$, then $X$ is a hypergraph. An isomorphism between two combinatorial objects $X=(H, E)$ and $Y=\left(H^{\prime}, E^{\prime}\right)$ is a bijection $\delta: H \rightarrow H^{\prime}$ such that $\delta(E)=E^{\prime}$. An automorphism of a combinatorial object $X$ is an isomorphism from $X$ to itself. Let $G$ be a group and $X=(G, E)$ a combinatorial object. Define $g_{L}: G \rightarrow G$ by $g_{L}(h)=g h$ and let $G_{L}=\left\langle g_{L}: g \in G\right\rangle$. Then $X$ is a Cayley object of $G$ if and only if $G_{L} \leq \operatorname{Aut}(X)$. A Cayley object $X$ of $G$ is a CI-object of $G$ if and only if whenever $X^{\prime}$ is a Cayley object of $G$ isomorphic to $X$, then some $\alpha \in \operatorname{Aut}(G)$ is an isomorphism from $X$ to $X^{\prime}$. Similarly, $G$ is a CI-group with respect to $\mathcal{K}$ if and only if every Cayley object in the class of combinatorial objects $\mathcal{K}$ is a CI-object of $G$, and a $C I$-group if $G$ is a CI-group with respect to every class $\mathcal{K}$ of combinatorial objects. It is known [31] that $G$ is a CI-group if and only if $|G|=4$ or $G \cong \mathbb{Z}_{n}$, with $\operatorname{gcd}(n, \varphi(n))=1$. Hence neither $\mathbb{Z}_{p^{2}}$ nor $\mathbb{Z}_{p}^{2}$ is a CI-group unless $p=2$, although $\mathbb{Z}_{p}^{2}$ is a 
CI-group with respect to graphs [13]. We begin with a characterization of when two Cayley objects of a $p$-group $G$ can be isomorphic provided their automorphism groups share a common Sylow $p$-subgroup.

Lemma 15 Let $X$ and $Y$ be Cayley objects of a p-group $G$, and $P$ a Sylow p-subgroup of both $\operatorname{Aut}(X)$ and $\operatorname{Aut}(Y)$. Then $X$ and $Y$ are isomorphic if and only if there exists $\delta \in N_{S_{G}}(P)$ such that $\delta(X)=Y$.

Proof: $\quad \Rightarrow$ Let $\omega: X \rightarrow Y$ be an isomorphism. Then $\omega^{-1} P \omega \subset \operatorname{Aut}(X)$, and $\omega^{-1} P \omega \leq$ $P_{1}$, a Sylow $p$-subgroup of $\operatorname{Aut}(X)$. Hence there exists $\beta \in \operatorname{Aut}(X)$ such that $\beta^{-1} P_{1} \beta=P$, so that $\beta^{-1} \omega^{-1} P \omega \beta \leq P$, which means $\omega \beta \in N_{S_{G}}(P)$. Furthermore, $\omega \beta: X \rightarrow Y$ is an isomorphism.

Corollary 1 Let $X$ and $Y$ be Cayley objects of $\mathbb{Z}_{p^{2}}$, such that $P_{i}$ is a Sylow p-subgroup of both $\operatorname{Aut}(X)$ and $\operatorname{Aut}(Y)$, for some $2 \leq i \leq p-1$. Let $\beta \in \mathbb{F}_{p}^{*}$ such that $|\beta|=p-1$. Then $X$ and $Y$ are isomorphic if and only of they are isomorphic by $\alpha=\hat{\beta}^{j} \gamma_{i+1}^{k}$, for some $1 \leq j \leq p-1$ and $1 \leq k \leq p$.

Proof: $\quad \Rightarrow$ From Lemmas 15 and 5, we know that $X$ and $Y$ are isomorphic if and only if they are isomorphic by some $\delta \in N_{S_{p^{2}}}\left(P_{i}\right)=\left\langle N_{S_{p^{2}}}(\langle\tau\rangle), \gamma_{i+1}\right\rangle$. As $P_{i} \triangleleft N_{S_{p^{2}}}\left(P_{i}\right)$ and $\left|N_{S_{p^{2}}}\left(P_{i}\right) / P_{i}\right|=(p-1) p$, there are $(p-1) p$ cosets of $P_{i}$ in $N_{S_{p^{2}}}\left(P_{i}\right)$. As $\hat{\beta}, \gamma_{i+1} \notin P_{i}$, these $(p-1) p$ cosets are $P_{i} \beta^{j} \gamma_{i+1}^{k}, 1 \leq j \leq p-1$ and $1 \leq k \leq p$. Hence $\delta$ may be written in the form $\delta=g \alpha$, with $g \in P_{i}$ and $\alpha=\hat{\beta}^{j} \gamma_{i+1}^{k}$. Then $\alpha \in N_{S_{p^{2}}}\left(P_{i}\right)$ and, because $\delta(X)=Y$ and $g \in \operatorname{Aut}(Y)$, we have $\alpha(X)=Y$.

Corollary 2 Let $X$ and $Y$ be Cayley objects of $\mathbb{Z}_{p}^{2}$ with $\Pi_{1}$ a Sylow p-subgroup of $\operatorname{Aut}(X)$ and $\Pi_{2}$ a Sylow p-subgroup of $Y$. Let $\alpha_{1} \in \operatorname{Aut}\left(\mathbb{Z}_{p}^{2}\right)$ such that $\alpha_{1} \Pi_{1} \alpha_{1}^{-1}=P_{i}^{\prime}$ and $\alpha_{2} \in$ $\operatorname{Aut}\left(\mathbb{Z}_{p}^{2}\right)$ such that $\alpha_{2} \Pi_{2} \alpha_{2}^{-1}=P_{i}^{\prime}, 1 \leq i \leq p-1$. Let $\beta \in \mathbb{F}_{p}^{*}$ such that $|\beta|=p-1$. Then $X$ and $Y$ are isomorphic if and only if they are isomorphic by $\alpha_{1} \bar{\beta}^{j} \tilde{\beta}^{k} \gamma_{i+1}^{\ell} \alpha_{2}^{-1}, 1 \leq j$, $k \leq p-1,1 \leq \ell \leq p$.

Proof: Note that $P_{i}$ is a Sylow $p$-subgroup of both $\operatorname{Aut}\left(\alpha_{1}(X)\right)$ and $\operatorname{Aut}\left(\alpha_{2}(Y)\right)$. It follows then by arguments analogous to those in Corollary 1 that $\alpha_{1}(X)$ and $\alpha_{2}(Y)$ are isomorphic if and only if they are isomorphic by some $\omega \in N_{S_{p^{2}}}\left(P_{i}^{\prime}\right)$. The result follows.

We remark that the case $P=P_{2}^{\prime}$ was considered in [4].

\subsection{Automorphism groups of Cayley graphs of $\mathbb{Z}_{p}^{2}$}

Using Theorem 4 we can calculate the full automorphism group of any vertex-transitive graph of order $p^{2}$. We actually will prove this result in slightly more generality, determining all 2-closed groups $G$ that contain a regular subgroup isomorphic to $\mathbb{Z}_{p}^{2}$ (as was done in the previously cited paper). We remark that Klin and Pöschel [25] have already calculated the full automorphism groups of circulant graphs of order $p^{k}$ (that is, of Cayley graphs of $\mathbb{Z}_{p^{k}}$ ). 
Theorem 14 Let $G$ be a 2-closed subgroup of $S_{p^{2}}$ such that $G$ contains the left regular representation of $\mathbb{Z}_{p}^{2}$.

1. If $G$ is doubly transitive, then $G=S_{p^{2}}$.

2. If $G$ is simply primitive and solvable, then $G \leq \operatorname{AGL}(2, p)$.

3. If $G$ is simply primitive and nonsolvable, then $G \leq A G L(2, p)$ or $G=S_{2}$ 2 $S_{p}$ in its product action.

4. If $G$ is imprimitive, solvable, and has elementary abelian Sylow p-subgroup, then either $G<\operatorname{AGL}(1, p) \times \operatorname{AGL}(1, p)$ or $G=S_{3} \times S_{3}$ (and $p=3$ ).

5. If $G$ is imprimitive, nonsolvable, and has elementary abelian Sylow $p$-subgroup, then either $G=S_{p} \times S_{p}$ or $G=S_{p} \times A$, where $A<\operatorname{AGL}(1, p)$.

6. If $G$ is imprimitive with Sylow $p$-subgroup of order at least $p^{3}$, then $G=G_{1} 2 G_{2}$, where $G_{1}$ and $G_{2}$ are 2-closed permutation groups of degree $p$.

Proof: (1) If $G$ is doubly transitive, then clearly $G=S_{p^{2}}$.

(5) If $G$ is imprimitive, nonsolvable, and has elementary abelian Sylow $p$-subgroup, then by Theorem 4, we have that $G=\left\{(\sigma, \tau) \in H \times N_{S_{p}}(K): f(\sigma) \in \tau K\right\}$, where $K, H \leq S_{p}$ and $f: H \rightarrow N_{S_{p}}(K) / K$ is a group homomorphism.

Let $\tau_{1} \in H$ be a $p$-cycle and $\tau_{2} \in K$ be a $p$-cycle. Then $\left(\tau_{1}, 1_{S_{p}}\right) \in G$ and $\left(1_{S_{p}}, \tau_{2}\right) \in G$. Furthermore, $G$ admits complete block systems $\mathcal{B}_{1}$ and $\mathcal{B}_{2}$ of $p$ blocks of cardinality $p$ formed by the orbits of $\left.\left\langle\left(\tau_{1}, 1_{S_{p}}\right)\right)\right\rangle$ and $\left\langle\left(1_{S_{p}}, \tau_{2}\right)\right\rangle$, respectively (because $\left.G \leq S_{p} \times S_{p}\right)$.

If both $\operatorname{fix}_{G}\left(\mathcal{B}_{1}\right)=\left\{\left(\delta, 1_{S_{p}}\right): \delta \in \operatorname{Ker}(f)\right\}$ and $\left.\operatorname{fix}_{G}\left(\mathcal{B}_{2}\right)=\left\{\left(1_{S_{p}}\right), \gamma\right): \gamma \in K\right\}$ are solvable, then $\operatorname{fix}_{G}\left(\mathcal{B}_{1}\right) \leq \operatorname{AGL}(1, p)$ and $\operatorname{fix}_{G}\left(\mathcal{B}_{2}\right) \leq \operatorname{AGL}(1, p)$. Then $K \leq \operatorname{AGL}(1, p)$ and $N_{S_{p}}(K)=\operatorname{AGL}(1, p)$ is solvable. Hence both $\operatorname{Ker}(f)$ and $f(H)$ are solvable so that $H$ is solvable. Thus $G$ is solvable, a contradiction.

We now know that either $\operatorname{fix}_{G}\left(\mathcal{B}_{1}\right)$ or $\operatorname{fix}_{G}\left(\mathcal{B}_{2}\right)$ is nonsolvable. We will show that if $\operatorname{fix}_{G}\left(\mathcal{B}_{2}\right)$ is doubly transitive (which includes the nonsolvable case), then $G=H \times S_{p}$. The case where $\operatorname{fix}_{G}\left(\mathcal{B}_{1}\right)$ is nonsolvable is handled in a similar fashion.

If $\operatorname{fix}_{G}\left(\mathcal{B}_{2}\right)$ is nonsolvable, then by Theorem $\left.1 \mathrm{fix}_{G}\left(\mathcal{B}_{2}\right)\right|_{B}$ is doubly transitive for every $B \in \mathcal{B}_{2}$. Hence $\operatorname{Stab}_{\mathrm{fix}_{G}\left(\mathcal{B}_{2}\right)}(i, j) \neq 1$ for every $(i, j) \in \mathbb{Z}_{p}^{2}$. Define an equivalence relation $\equiv$ on $\mathbb{Z}_{p}^{2}$ by $(i, j) \equiv(k, \ell)$ if and only if $\operatorname{Stab}_{\mathrm{fix}_{G}\left(\mathcal{B}_{2}\right)}(i, j)=\operatorname{Stab}_{\mathrm{fix}_{G}\left(\mathcal{B}_{2}\right)}(k, \ell)$. As $G \leq S_{p} \times S_{p}$, there are $p$ equivalence classes of $\equiv$ and each equivalence class of $\equiv$ contains exactly one element from each block of $\mathcal{B}_{2}$. As $\left.\operatorname{fix}_{G}\left(\mathcal{B}_{2}\right)\right|_{B}$ is doubly transitive, $\left.\operatorname{Stab}_{\text {fix }_{G}\left(\mathcal{B}_{2}\right)}(i, j)\right|_{B}$ has two orbits for every $B \in \mathcal{B}_{2}$. One orbit consists of $\{(k, \ell)\}$, where $(k, \ell) \equiv(i, j)$ and the other consisting of the remaining elements of the block $B^{\prime}$ of $\mathcal{B}_{2}$ that contains $(k, \ell)$. Let $\Gamma$ be an orbital digraph of $G$ with $((i, j),(k, \ell)) \in E(\Gamma)$. If $i=k$, then $\Gamma=p K_{p}$ (the union of $p$ disjoint copies of $K_{p}$ ) and so $\operatorname{Aut}(\Gamma)=S_{p}$ ? $S_{p}$. If $i \neq j$, then, as $\Gamma$ is an orbital digraph, either $(i, j)$ is only adjacent to $(k, \ell)$ or $(i, j)$ is adjacent to every element of $B^{\prime}$ except $(k, \ell)$. In either case, it is straightforward to verify that $\left\{\left(1_{S_{p}}, \gamma\right): \gamma \in S_{p}\right\} \leq \operatorname{Aut}(\Gamma)$. As $G$ is the intersection of the automorphism group of all orbital digraphs of $G$, we have $K=S_{p}, N_{S_{p}}(K)=S_{p}$ and $f=1$. Thus $G=H \times K=H \times S_{p}$ as required. Thus either $H<\operatorname{AGL}(1, p)$ or $H$ is doubly transitive (as $\operatorname{AGL}(1, p)$ is doubly transitive). Analogous arguments will then show that if $H$ is doubly transitive, then $H=S_{p}$. Thus (5) follows.

(4) If $G$ is imprimitive, solvable, and has elementary abelian Sylow $p$-subgroup, then we may define $H, K$, and $f$ as in Theorem 4 . Both $H$ and $N_{S_{p}}(K)$ are solvable, so that 
$K$ is solvable and, by Theorem 1 , we have $H, K \leq \operatorname{AGL}(1, p)$. As $N_{S_{p}}(\operatorname{AGL}(1, p))=$ $\operatorname{AGL}(1, p)$, we have that $G \leq \operatorname{AGL}(1, p) \times \operatorname{AGL}(1, p)$. As $\operatorname{AGL}(1, p)$ is itself doubly transitive, if $G=\operatorname{AGL}(1, p) \times \operatorname{AGL}(1, p)$ then $\left.\operatorname{fix}_{G}(\mathcal{B})\right|_{B}$ is doubly transitive for every complete block system $\mathcal{B}$ of $G$ and every block $B \in \mathcal{B}$. It then follows by arguments above that $\operatorname{fix}_{G}(\mathcal{B}) \cong S_{p}$, a contradiction unless $p=3$. If $p=3$, then $\operatorname{AGL}(1, p) \times \operatorname{AGL}(1, p)=$ $S_{3} \times S_{3}$, a group listed in (4). Thus (4) follows.

$(2,3)$ If $G$ is simply primitive, then by Theorem $4, G$ has an elementary abelian Sylow $p$-subgroup and either $G \leq \operatorname{AGL}(2, p)$ or $G$ contains an imprimitive subgroup $H$ of index 2 . If $G \leq \operatorname{AGL}(2, p)$, then the result follows, so we may assume $G$ contains an imprimitive subgroup $H$ of index 2 . Note that $G$ is solvable if and only if $H$ is solvable.

If $H$ is solvable, then $H$ has an elementary abelian Sylow $p$-subgroup, and so $G$ has an elementary abelian Sylow $p$-subgroup. Furthermore, $G$ is solvable. Let $N$ be a minimal normal subgroup of $G$. Then $N$ is an elementary abelian $q$-group for some prime $q$. As $G$ is primitive, $N$ is transitive, $q=p$ and $|N|=p^{2}$. Thus $G \leq N_{S_{\mathbb{Z}_{p} \times \mathbb{Z}_{p}}}(N) \leq \operatorname{AGL}(2, p)$ and (2) follows.

If $H$ is nonsolvable, then by (5) proven above and the fact that if $H \leq G$, then $\operatorname{cl}(H) \leq$ $\operatorname{cl}(G)$, we have that either $H=S_{p} \times S_{p}$ or $H=A \times S_{p}$, with $A<\operatorname{AGL}(1, p)$. It then follows by [10, Theorem 4.6A] that $G=S_{2} 2 S_{p}$ with the product action. Thus (3) follows.

(6) If $G$ has a Sylow $p$-subgroup $P$ of order at least $p^{3}$, then $P$ admits a complete block system $\mathcal{B}$ of $p$ blocks of cardinality $p$. Then $\left|\operatorname{fix}_{P}(\mathcal{B})\right| \geq p^{2}$ so that $\operatorname{Stab}_{\operatorname{fix}_{P}(\mathcal{B})}(0,0) \neq 1$. As $\operatorname{fix}_{P}(\mathcal{B})$ is a $p$-group, we have that if $\gamma \in \operatorname{Stab}_{\text {fix }_{P}(\mathcal{B})}(0,0)$, then $\gamma$ fixes every point of the block of $\mathcal{B}$ that contains $(0,0)$. Define an equivalence relation $\equiv^{\prime}$ on $\mathbb{Z}_{p}^{2}$ by $(i, j) \equiv^{\prime}(k, \ell)$ if and only if $\operatorname{Stab}_{\operatorname{fix}_{P}(\mathcal{B})}(i, j)=\operatorname{Stab}_{\operatorname{fix}_{P}(\mathcal{B})}(k, \ell)$. It follows by comments above and the fact that $\operatorname{Stab}_{\operatorname{fix}_{P}(\mathcal{B})}(i, j)=\operatorname{Stab}_{P}(0,0)$, that the cardinality of each equivalence class of $\equiv^{\prime}$ is a multiple of $p$. It is straightforward to verify that the equivalence classes of $\equiv^{\prime}$ are blocks of $P$ so that each equivalence class of $\equiv^{\prime}$ has order $p$. Thus the equivalence classes of $\equiv^{\prime}$ form the complete block system $\mathcal{B}$. For convenience, we assume without loss of generality that $\mathcal{B}=\left\{\left\{(i, j): j \in \mathbb{Z}_{p}\right\}: i \in \mathbb{Z}_{p}\right\}$.

Let $\Gamma$ be an orbital digraph of $G$, with $P^{\prime}$ a Sylow $p$-subgroup of $\operatorname{Aut}(\Gamma)$ that contains $P$. Then $P^{\prime}$ admits $\mathcal{B}$ as a complete block system as well. If $\Gamma$ is disconnected, then $\Gamma=p \Gamma_{2}$, where $p \Gamma_{2}$ is the disjoint union of $p$ copies of the directed graph $\Gamma_{2}$ so that $\operatorname{Aut}(\Gamma)=S_{p}$ 2 Aut $\left(\Gamma_{2}\right)$. If $\Gamma$ is connected, let $((i, j),(k, \ell)) \in E(\Gamma)$ such that $i \neq k$. Then $(i, j) \not \equiv^{\prime}(k, \ell)$ so that there exists $\gamma \in P$ such that $\gamma(i, j)=(i, j)$ but $\gamma(k, \ell) \neq(k, \ell)$. Then $\gamma$ permutes the $p$ elements of $\left\{(k, m): m \in \mathbb{Z}_{p}\right\}$ as a $p$-cycle. We conclude that $((i, j),(k, m)) \in E(\Gamma)$ for every $m \in \mathbb{Z}_{p}$. As $\left.\operatorname{fix}_{P}(\mathcal{B})\right|_{B}$ is semiregular, where $B \in \mathcal{B}$ such that $(i, j) \in B$, we have that $((i, n),(k, m)) \in E(\Gamma)$ for every $n, m \in \mathbb{Z}_{p}$. Thus $\Gamma=\Gamma_{1} \imath \Gamma_{2}$ where $\Gamma_{1}$ and $\Gamma_{2}$ are digraphs of order $p$. It follows by [32, Theorem 1] that $\operatorname{Aut}(\Gamma)=$ $\operatorname{Aut}\left(\Gamma_{1}\right)$ 2 $\operatorname{Aut}\left(\Gamma_{2}\right)$ (although the cited theorem is stated only for graphs, it works as well for digraphs). $\mathrm{As} \operatorname{cl}(G)$ is the intersection of the automorphism groups of all orbital digraphs of $G$, we conclude that $G=G_{1}$ 乙 $G_{2}$ for 2 -closed groups $G_{1}, G_{2}$ of degree $p$. Thus (6) holds.

Theorem 15 Let $G$ be a 2-closed subgroup of $S_{p^{2}}$ that contains the left regular representation of $\mathbb{Z}_{p^{2}}$. Then one of the following is true: 
1. $G=S_{p^{2}}$,

2. $G \leq N_{S_{p^{2}}}\left(\left(\mathbb{Z}_{p^{2}}\right)_{L}\right)$,

3. $G=G_{1} 2 G_{2}$, where $G_{1}$ and $G_{2}$ are 2-closed groups of degree $p$.

Proof: If $G$ is doubly transitive, then $G=S_{p^{2}}$. Otherwise, as $\mathbb{Z}_{p^{2}}$ is a Burnside group [33, Theorem 25.3], $G$ is imprimitive. By Theorem 4, either a Sylow $p$-subgroup of $G$ is normal in $G$, or a Sylow $p$-subgroup is isomorphic to $\mathbb{Z}_{p} 2 \mathbb{Z}_{p}$. By arguments in Theorem 14 , if a Sylow $p$-subgroup of $G$ has order at least $p^{3}$, then $G=G_{1} 2 G_{2}$, where $G_{1}$ and $G_{2}$ are 2-closed groups of degree $p$. The result then follows.

Definition 16 A Cayley digraph $\Gamma$ of a group $G$ is normal if the left regular representation of $G$ is normal in $\operatorname{Aut}(\Gamma)$.

In [35, Problem 3], Ming-Yao Xu posed the problem of determining all nonnormal Cayley graphs of order $p^{2}$. We are now in a position to solve this problem.

Corollary 3 A Cayley digraph $\Gamma$ of a group of order $p^{2}$ is nonnormal if and only if $\Gamma$ is isomorphic to one of the following graphs.

1. $\Gamma=K_{p^{2}}, p \geq 3$, or $p=2$ and $G=\mathbb{Z}_{4}$,

2. $\Gamma=\Gamma_{1} 2 \Gamma_{2}$, where $\Gamma_{1}$ and $\Gamma_{2}$ are Cayley digraphs of the cyclic group of order $p, p \geq 3$,

3. $\Gamma$ is a Cayley digraph of $\mathbb{Z}_{p}^{2}$ but not $\mathbb{Z}_{p^{2}}, p \geq 5$, with connection set $S=\{(i, 0),(0, j)$ : $\left.i, j \in \mathbb{Z}_{p}\right\}$ or the complement of this graph,

4. $\Gamma$ is a Cayley digraph of $\mathbb{Z}_{p}^{2}$ but not $\mathbb{Z}_{p^{2}}, p \geq 5$, whose connection set $S$ satisfies the following properties, where $H=\left\{(0, i): i \in \mathbb{Z}_{p}\right\}$,

(A) $H \cap S=\emptyset$ or $H \cap S=H-\{(0,0)\}$,

(B) for every coset $(a, 0)+H \neq H$ of $H,((a, 0)+H) \cap S=(a, b)+H, \emptyset,\{(a, 0)\}$, or $((a, 0)+H)-\{(a, 0)\}$.

Proof: Let $G=\operatorname{Aut}(\Gamma)$. Then $\Gamma$ is normal if (2), (4) of Theorem 14 hold or (2) of Theorem 15 hold.

If either (1) of Theorem 14 or (1) of Theorem 15 holds, then $\operatorname{Aut}(\Gamma)=S_{p^{2}}$ and $\Gamma$ is not normal unless $p=2$, in which case the left regular representation of $\mathbb{Z}_{2}^{2}$ is a normal subgroup of $S_{4}$ but the left regular representation of $\mathbb{Z}_{4}$ is not a normal subgroup of $S_{4}$ and (1) follows.

If (6) of Theorem 14 or (3) of 15 holds, then $\Gamma=\Gamma_{1} 2 \Gamma_{2}$ where $\Gamma_{1}$ and $\Gamma_{2}$ are Cayley digraphs of $\mathbb{Z}_{p}$. It is then straightforward to verify, as $\operatorname{Aut}(\Gamma)=\operatorname{Aut}\left(\Gamma_{1}\right) \prec \operatorname{Aut}\left(\Gamma_{2}\right)$ that left regular representations of $\mathbb{Z}_{p^{2}}$ and $\mathbb{Z}_{p}^{2}$ are not normal in $\operatorname{Aut}(\Gamma)$ unless $p=2$. Whence (2) holds. We conclude that the remaining nonnormal Cayley digraphs must be Cayley digraphs of $\mathbb{Z}_{p}^{2}$ but not $\mathbb{Z}_{p^{2}}$.

If (3) of Theorem 14 holds, then (3) follows by [35, Theorem 2.12].

Finally, if (5) of Theorem 14 holds, $\Gamma$ will be nonnormal provided that $p \geq 5$. Further, $\operatorname{Aut}(\Gamma)$ admits a complete block system $\mathcal{B}$ of $p$ blocks of cardinality $p$, which we may assume (by replacing $\Gamma$ with its image under an appropriate automorphism of $\mathbb{Z}_{p}^{2}$ ) that $\mathcal{B}$ is formed by the orbits of $H_{L}$ and that $\left.\operatorname{fix}_{\mathrm{Aut}(\Gamma)}(\mathcal{B})\right|_{B}=S_{p}$ for every $B \in \mathcal{B}$. As fix $\left.\operatorname{Aut}_{\mathrm{Au}(\Gamma)}(\mathcal{B})\right|_{B}=S_{p}$, we have that $\Gamma[H]=K_{p}$ or $\bar{K}_{p}$, the complete graph on $p$ vertices or its complement. Whence $H \cap S=\emptyset$ or $H \cap S=H-\{(0,0)\}$. Define an equivalence relation $\equiv$ on $\mathbb{Z}_{p}^{2}$ by $(i, j) \equiv$ 
$(k, \ell)$ if and only if $\operatorname{Stab}_{\text {fix }_{\mathrm{Aut}(\Gamma)}(\mathcal{B})}(i, j)=\operatorname{Stab}_{\mathrm{fix}_{\mathrm{Aut}(\Gamma)}(\mathcal{B})}(k, \ell)$. It is then straightforward to verify that there are $p$ equivalence classes of $\equiv$ and that these $p$ equivalence classes of $\equiv$ form a complete block system $\mathcal{C}$ of $\operatorname{Aut}(\Gamma)$. Again, if necessary, we replace $\Gamma$ with its image under an appropriate automorphism of $\mathbb{Z}_{p}^{2}$ and assume that $\mathcal{B}$ is formed by the orbits of $H_{L}$ and $\mathcal{C}=\left\{\left\{(i, j): i \in \mathbb{Z}_{p}\right\}: j \in \mathbb{Z}_{p}\right\}$. Let $a \in \mathbb{Z}_{p}^{*}$. Then $(0,0)$ is adjacent to either: no vertex of $(a, 0)+H$; every vertex of $(a, 0)+H$; only the vertex of $(a, 0)$ of $(a, 0)+H$; or every vertex of $(a, 0)+H$ except $(a, 0)$. Thus (4) follows.

The converse is straightforward.

\section{Acknowledgments}

This research was partially supported by grants from the National Science Foundation (DMS-9623256 and DMS-9801136).

We thank the anonymous referee for numerous suggestions that improved the exposition.

\section{References}

1. B. Alspach and T.D. Parsons, "Isomorphism of circulant graphs and digraphs," Discrete Math. 25(2) (1979), 97-108.

2. L. Babai, "Isomorphism problem for a class of point-symmetric structures," Acta Math. Sci. Acad. Hung. 29 (1977), 329-336.

3. M. Bardoe and P. Sin, "The permutation modules for $\operatorname{GL}\left(n+1, \mathbb{F}_{q}\right)$ acting on $\mathbb{P}^{n}\left(\mathbb{F}_{q}\right)$ and $\mathbb{F}_{q}^{n+1}$," J. London Math. Soc. 61(1) (2000), 58-80.

4. N. Brand, "Quadratic isomorphism of $\mathbb{Z}_{p} \times \mathbb{Z}_{p}$ objects," Congr. Numer. 58 (1987), 157-163.

5. W. Burnside, "On some properties of groups of odd order," J. London Math. Soc. 33 (1901), 162-185.

6. W. Burnside, Theory of Groups of Finite Order, Cambridge University Press, Cambridge, 1911.

7. M. Burrow, Representation Theory of Finite Groups, Dover, New York, 1993.

8. P.J. Cameron, "Finite permutation groups and finite simple groups," Bull. London Math. Soc. 13 (1981), 1-22.

9. H. Cartan and S. Eilenberg, Homological Algebra, Princeton University Press, Princeton, 1956.

10. J.D. Dixon and B. Mortimer, Permutation Groups, Springer-Verlag, New York, Berlin, Heidelberg, Graduate Texts in Mathematics, Vol. 163, 1996.

11. E. Dobson, "Isomorphism problem for Cayley graphs of $\mathbb{Z}_{p}^{3}$," Discrete Math. 147 (1995), 87-94.

12. W. Feit, "Some consequences of the classification of finite simple groups," in The Santa Cruz Conference on Finite Groups, B. Cooperstein and G. Mason (Eds.), Amer. Math. Soc., Providence, 1980. Proc. Symp. Pure Math. 37 (1980), 175-181.

13. C.D. Godsil, "On Cayley graph isomorphisms," Ars Combin. 15 (1983), 231-246.

14. D. Gorenstein, Finite Groups, Chelsea, New York, 1980.

15. R.M. Guralnick, "Subgroups of prime power index in a simple group," J. of Algebra 81 (1983), 304-311.

16. C. Hering, "Transitive linear groups and linear groups which contain irreducible subgroups of prime order II," J. Algebra 93(1) (1985), 151-164.

17. W.C. Huffman, "The equivalence of two cyclic objects on $p q$ elements," Discrete Math. 154 (1996), 103-127.

18. W.C. Huffman, "Codes and Groups," in Handbook of Coding Theory, V.S. Pless and W.C. Huffman (Eds.), Vol. 2, Elsevier, 1998, pp. 1345-1440.

19. W.C. Huffman, V. Job, and V. Pless, "Multipliers and generalized multipliers of cyclic objects and cyclic codes," J. Combin. Theory Ser. A 62 (1993), 183-215.

20. B. Huppert, "Zweifach transitive, auflösbare Permutationsgruppen,” Math. Z. 68 (1957), 126-150.

21. T. Hungerford, Algebra, Holt, Rinehart and Winston, 1974.

22. G.A. Jones, "Abelian subgroups of simply primitive groups of degree $p^{3}$, where $p$ is prime," Quart. J. Math. Oxford 30(2) (1979), 53-76. 
23. M. Klemm, "Über die Reduktion von Permutationsmoduln," Math. Z. 143 (1975), 113-117.

24. A.S. Kleshchev and A.A. Premet, "On second degree cohomology of symmetric and alternating groups," Comm. Alg. 21(2) (1993), 583-600.

25. M.Ch. Klin and R. Pöschel, "The isomorphism problem for circulant graphs with $p^{n}$ vertices," Preprint P-34/80 ZIMM, Berlin, 1980

26. W. Knapp and P. Schmid, "Codes with prescribed permutation group," J Algebra 67 (1980), 415-435.

27. F.J. MacWilliams and M.J.A. Sloane, The Theory of Error Correcting Codes, North-Holland, New York, 1977.

28. D. Marušič and R. Scapellato, "Characterizing vertex transitive $p q$-graphs with imprimitive automorphism group," J. Graph Theory 16 (1992), 375-387.

29. B. Mortimer, "The modular permutation representations of the known doubly transitive groups," Proc. London Math. Soc. 41(3) (1980), 1-20.

30. O. Ore, "Contributions to the theory of groups of finite orders," Duke Math. J. 5 (1954), 431-460.

31. P.P. Pálfy, "Isomorphism problem for relational structures with a cyclic automorphism," Europ. J. Comb. 8 (1987), 35-43.

32. G. Sabidussi, "The lexicographic product of graphs," Duke Math. J. 28 (1961), 573-578.

33. H. Wielandt, Finite Permutation Groups, Academic Press, New York, 1964.

34. H. Wielandt, Permutation Groups Through Invariant Relations and Invariant Functions, Lecture Notes, Ohio State University, 1969.

35. M.Y.Xu, “Automorphism groups and isomorphisms of Cayley digraphs,” Discrete Math. 182 (1998), 309-319. 